\title{
Generalized Brans-Dicke theories in light of evolving dark energy
}

\author{
Alex Zucca $\odot,{ }^{1}$ Levon Pogosian $\odot,{ }^{1,2}$ Alessandra Silvestri, ${ }^{3}$ Yuting Wang, ${ }^{4}$ and Gong-Bo Zhao ${ }^{4,5,2}$ \\ ${ }^{1}$ Department of Physics, Simon Fraser University, Burnaby, British Columbia V5A 1S6, Canada \\ ${ }^{2}$ Institute of Cosmology and Gravitation, University of Portsmouth, \\ Portsmouth PO1 3FX, United Kingdom \\ ${ }^{3}$ Institute Lorentz, Leiden University, P.O. Box 9506, Leiden 2300 RA, Netherlands \\ ${ }^{4}$ National Astronomy Observatories, Chinese Academy of Sciences, \\ Beijing 100101, People's Republic of China \\ ${ }^{5}$ School of Astronomy and Space Science, University of Chinese Academy of Sciences, \\ Beijing 100049, People's Republic of China
}

(Received 19 July 2019; accepted 30 January 2020; published 13 February 2020)

\begin{abstract}
The expansion history of the Universe reconstructed from a combination of recent data indicates a preference for a changing dark energy (DE) density. Moreover, the DE density appears to be increasing with cosmic time, with its equation of state being below -1 on average, and possibly crossing the so-called phantom divide. Scalar-tensor theories, in which the scalar field mediates a force between matter particles, offer a natural framework in which the effective DE equation of state can be less than -1 and cross the phantom barrier. We consider the generalized Brans-Dicke (GBD) class of scalar-tensor theories and reconstruct their Lagrangian given the effective DE density extracted from recent data. Then, given the reconstructed Lagrangian, we solve for the linear perturbations and investigate the characteristic signatures of these reconstructed GBD in the cosmological observables, such as the cosmic microwave background $(\mathrm{CMB})$ anisotropy, the galaxy number counts, and their cross-correlations. In particular, we demonstrate that the integrated Sachs-Wolfe effect probed by the cross-correlation of CMB with the matter distribution can rule out scalar-tensor theories as the explanation of the observed DE dynamics independently from the laboratory and Solar System fifth force constraints.
\end{abstract}

DOI: 10.1103/PhysRevD.101.043518

\section{INTRODUCTION}

The observed accelerated expansion of the Universe has been puzzling cosmologists since its discovery two decades ago $[1,2]$. Within the context of general relativity (GR), it implies the existence of an energy-momentum component with a negative equation of state (EOS), referred to as dark energy (DE). The standard cosmological model, $\Lambda \mathrm{CDM}$, in which DE is the constant energy of the vacuum, provides a good fit to a plethora of cosmological observations such as the cosmic microwave background (CMB) anisotropies [3,4], baryon acoustic oscillations (BAO) [5-7], type Ia supernovae [8,9], galaxy clustering [10] and galaxy lensing $[11,12]$. However, $\Lambda \mathrm{CDM}$ is not fully satisfactory from the theoretical perspective, as the observed value of the vacuum energy requires an extreme fine-tuning of the cosmological constant $\Lambda$ in the context of the present understanding of particle interactions [13]. Also, with the data becoming more accurate, several "tensions" between different datasets have begun to arise when interpreting observations within the $\Lambda \mathrm{CDM}$ model $[4,14-17]$. Although these tensions might just be due to unaccounted systematic effects or rare statistical fluctuations [18], they generated significant interest in possible extensions of $\Lambda \mathrm{CDM}$ capable of relieving the tensions [19-26], including the possibility of the DE density evolving with time [27-30].

Using a combination of available observations, nonparametric reconstructions of the DE dynamics were performed in $[27,28]$. Interestingly, they show a preference for an increasing effective DE density, i.e., one with an EOS, $w_{\mathrm{DE}}^{\mathrm{eff}}<-1$. The reconstruction indicates a crossing of the socalled phantom divide [31-33] of $w_{\mathrm{DE}}^{\text {eff }}=-1$, also reported earlier studies, such as $[34,35]$. Such dynamics cannot be explained by a minimally coupled quintessence field DE but could be realized in scalar-tensor extensions of GR where the additional scalar field $\phi$ mediates a force between particles [32,36-38]. In fact, scalar-tensor theories possess enough freedom to reproduce any expansion history.

The aim of this paper is to investigate scalar-tensor theories of the generalized Brans-Dicke (GBD) type capable of realizing the expansion histories reconstructed in $[27,28]$. Using the observed $H(a)$ as input and making certain assumptions about the scalar field coupling function, we systematically scan the parameter space to reconstruct the GBD Lagrangians consistent with that $H(a)$. We then solve for the cosmological perturbations and calculate predictions for the CMB and galaxy power spectra and 
other observables to isolate the theories that are in agreement with current data.

Late time deviations from $\Lambda \mathrm{CDM}$ are mainly encoded in the CMB temperature through the integrated Sachs-Wolfe (ISW) effect. Although too small to be detected from the CMB temperature autocorrelation, the ISW contribution can be probed by cross-correlating the CMB temperature maps with the foreground galaxies number counts [39-42], which can be a useful probe for DE $[43,44]$. In $\Lambda$ CDM, the accelerating expansion results in decaying gravitational potentials, yielding a strictly positive ISW effect. In scalartensor theories, however, the ISW effect can have a positive or negative sign depending on whether the enhanced clustering due to the fifth force, which yields a negative ISW, dominates over the effect of the accelerating expansion $[45,46]$. We find that most of the GBD theories reconstructed in this work predict CMB-matter crosscorrelations that are significantly different from those in $\Lambda \mathrm{CDM}$ and, therefore, can be ruled out or confirmed with the next-generation galaxy surveys, such as DESI [47], LSST [48,49] and Euclid [50-52].

Our main points and results can be summarized as follows. In Sec. II we discuss the DE reconstruction results from [28] showing that observations appear to favor a timedependent DE density. There is some evidence for a nonmonotonic evolution and an overall increase of the DE density with time. As we show in Sec. III, such DE dynamics could, in principle, be realized in GBD-type scalar-tensor theories, where it would be a manifestation of the nonminimal coupling of the scalar field to matter. We note that, when constraining such theories, one should directly constrain the $\mathrm{DE}$ density, instead of the $\mathrm{DE}$ equation of state, as the latter can be singular. We then develop a formalism to systematically search for scalartensor theories capable of reproducing the observed expansion history. The GBD theories contain two free functions of the scalar field, the coupling function $F(\phi)$ and the potential $V(\phi)$. We consider two cases: model 1, in which $F(\phi)$ is a monotonic function of $\phi$, and model 2, in which it is a general function of the scale factor $a$. In both cases, we search the parameter space for GBD theories that are free of instabilities. We find that GBD theories with a monotonic coupling function cannot accommodate expansion histories in which the effective DE increased by a large fraction or is nonmonotonic. Allowing for a general coupling function $F(a)$ makes it possible to find viable GBD theories with a nonmonotonically evolving and increasing effective DE density. In Sec. IV, we evaluate the key cosmological observables predicted by the viable GBD theories that includes the CMB-galaxy cross-correlation spectra at three representative redshifts. We find that GBD theories capable of accommodating an increasing or a nonmonotonic DE density generically predict a sizable ISW signal at $z \sim 1$, which would be a robust way to rule out such GBD theories using cosmological datasets alone. We conclude with a summary in Sec. V.

\section{THE RECONSTRUCTED DARK ENERGY DENSITY}

A Bayesian, nonparametric reconstruction of the time evolution of the DE density was performed in [28] using the correlated prior method introduced in [53,54]. The effective DE energy density is modeled through the dimensionless function $X(a)$ that enters the Friedmann equation via

$$
H^{2}=H_{0}^{2}\left[E_{m}(a)+\Omega_{\Lambda} X(a)\right],
$$

where $E_{m} \equiv \sum_{i} \rho_{i}(a) / \rho_{\text {crit }}^{0}$ includes contributions of all matter and radiation fields, i.e., baryons, cold dark matter $(\mathrm{CDM})$, photons and neutrinos, and $X(a) \equiv \rho_{\mathrm{DE}}^{\text {eff }}(a) /$ $\rho_{\mathrm{DE}}^{\text {eff }}(a=1)$ is due to any contribution to the standard Friedmann equation from terms other than the matter and radiation. Solving for the cosmological perturbations would require making additional assumptions regarding the underlying DE or modified gravity theory $[55,56]$; hence, only observables probing the background expansion were used in [28] to keep the reconstruction model independent. The datasets included the CMB distance priors, the "joint lightcurve analysis" sample of supernovae type Ia (SNe Ia) [57], the Hubble parameter $H_{0}$ from [14], the observational Hubble parameters data [58], and the BAO distance measurements from (i) the 6dF Galaxy survey [6], (ii) SDSS DR7 main Galaxy sample [59], (iii) the tomographic BOSS DR12 [60,61], (iv) eBOSS DR14 quasar sample [62] and (v) the Lyman- $\alpha$ forest of BOSS DR11 quasars [63,64].

In the reconstruction, $X(a)$ was parametrized in terms of its values at $N=40$ points in $a$, i.e., $X_{i}=X\left(a_{i}\right)$, $i=1, \ldots, N$, with $a_{i}$ distributed uniformly in the interval $a \in[1,0.001]$. If $X_{i}$ were assumed to be independent, fitting them to data would yield large uncertainties, rendering the reconstruction useless. Moreover, treating the bins as completely independent is an unreasonably strong assumption as, in any specific theory, the effective DE density would be correlated at nearby points in $a$. Motivated by these considerations, the method of $[53,54]$ introduces a prior that correlates the neighboring bins. Specifically, each $X_{i}$ is a treated as a Gaussian random variable with values at different $a$ correlated according to a specified correlation function, $\xi(|\Delta a|)=\xi\left(\left|a-a^{\prime}\right|\right) \equiv\left\langle\left[X(a)-X^{\mathrm{fid}}(a)\right]\left[X\left(a^{\prime}\right)-X^{\mathrm{fid}}\left(a^{\prime}\right)\right]\right\rangle$. Here, $X^{\mathrm{fid}}(a)$ is a reference fiducial model, and the correlation function $\xi$ is chosen so that it is nonzero for $\left|a-a^{\prime}\right|$ below a given "correlation length" $a_{c}$ and approaches zero at larger separations. The form of $\xi$ was taken to be $[53,54]$

$$
\xi(|\Delta a|)=\frac{\xi(0)}{1+\left(|\Delta a| / a_{c}\right)^{2}},
$$

although, as demonstrated in [53], the details of the particular functional form are not essential as long as it has the rough shape that interpolates from 1 at $\Delta a=0$ to 0 at $\Delta a \gg a_{c}$. The parameter $\xi(0)$ sets the strength 

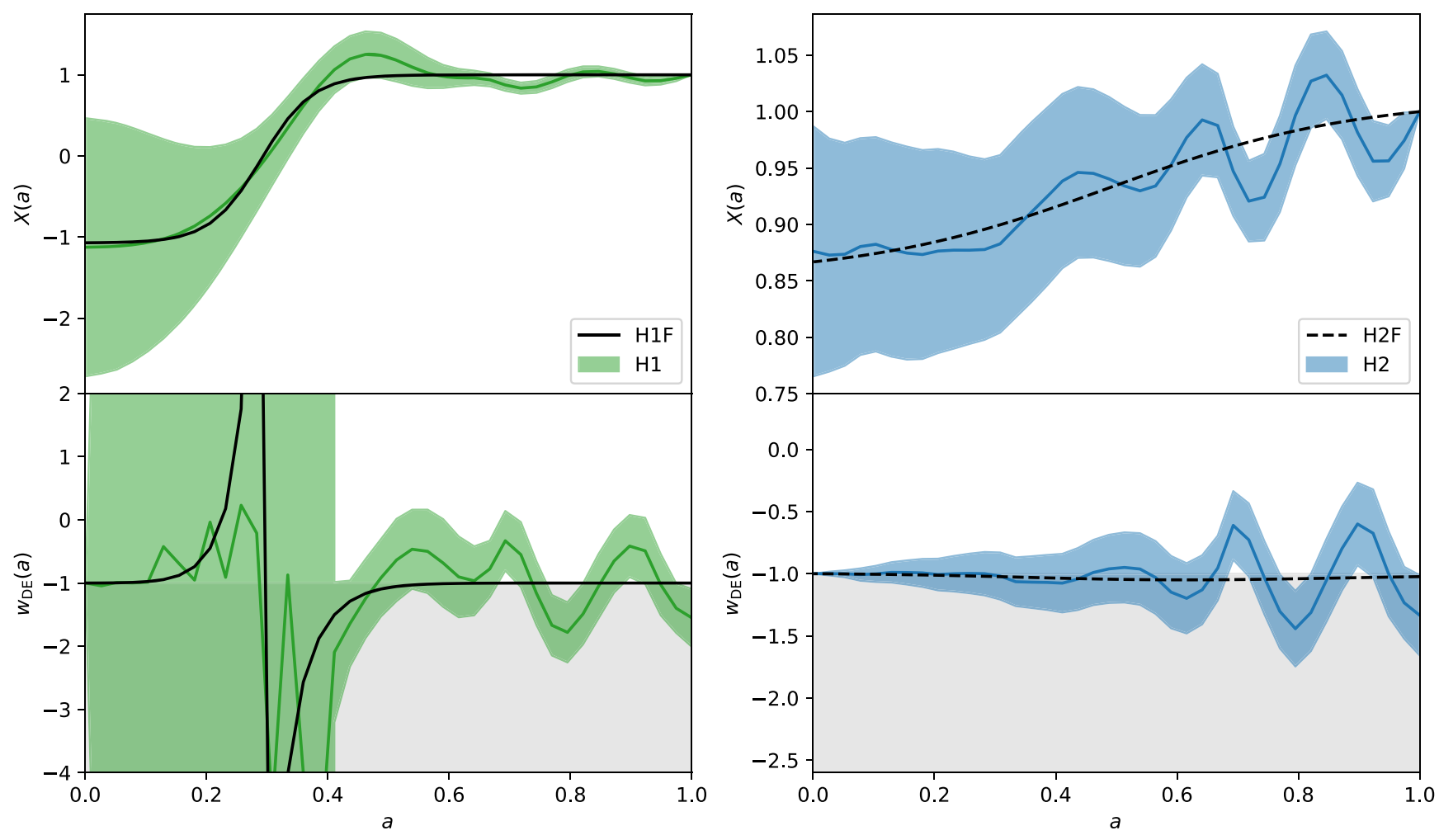

FIG. 1. The upper panels show the reconstructed normalized effective DE density $X(a)$, obtained using the standard prior (H1, left) and the evidence-weighted method (H2, right). Also shown are the corresponding hyperbolic tangent fits $\mathrm{H} 1 \mathrm{~F}$ (solid line) and $\mathrm{H} 2 \mathrm{~F}$ (dashed line). The lower panels show the corresponding effective DE equation of state.

of the prior and is related to the expected variance of the mean $\sigma_{\bar{X}}^{2}$ through $\sigma_{\bar{X}}^{2} \simeq \pi \xi(0) a_{c} /\left(a_{\max }-a_{\min }\right)$. The Gaussian prior effectively acts as an extra term in the total $\chi^{2}$, that is used to constrain the values of $X(a)$ in 40 bins in the interval $a \in[0.001,1]$.

The advantage of the correlated prior approach is that it allows one to control the strength of the prior and find the Bayesian evidence for each choice of the prior parameters. If the evidence for DE dynamics is larger than that for $\Lambda \mathrm{CDM}$ for a broad range of values of $a_{c}$ and $\sigma_{\bar{X}}$, i.e., does not require one to optimize them to improve the evidence, then one could say that dynamical DE is favored by observations. One can also define the evidence-weighted reconstruction, in which departures from $X(a)=1$ with low evidence get suppressed (see [28] for details).

Figure 1 shows the DE density reconstruction performed with the "standard" choice of the prior, $\sigma_{\bar{X}} \equiv 0.04, a_{c}=$ 0.06 (in green) along with the evidence-weighted reconstruction (in blue). They show two apparent trends: an overall increase in the effective DE density and an oscillatory behavior at $a \gtrsim 0.6$. The increase is driven by the local measurements of the Hubble constant $H_{0}$, whose larger value could be interpreted as an increase in DE density. The measurement of the BAO scale from the Lyman- $\alpha$ forest, which prefers a lower $H(z)$ at $z \sim 2.3$, further contributes to the same trend. ${ }^{1}$ Oscillations, on the other hand, are caused by the combination of the tomographic BAO and the JLA SNe Ia data which happen to have matching oscillatory patterns.

One can see that the apparently large deviation from $X(a)=1$ at high redshifts, seen in the standard reconstruction in Fig. 1, is not present in the evidenceweighted curve. The ability of data to constrain DE at $z>3$ is very weak and the reconstruction there is almost completely determined by the prior. This implies no Bayesian evidence for large deviations at high $z$, although the data still prefers a modest increase in DE density.

The lower panels in Fig. 1 show the corresponding effective DE EOS $w_{\mathrm{DE}}^{\mathrm{eff}}(a)$. They are obtained by generating an ensemble of $X(a)$ from its mean and the covariance

\footnotetext{
${ }^{1}$ The reconstruction in [28] was based on the Ly $\alpha \mathrm{BAO}$ analysis of BOSS DR11 quasars [64] that showed a $2.5 \sigma$ deviation from the best-fit $\Lambda \mathrm{CDM}$. The tension has since been reduced to $1.7 \sigma$ with the eBOSS DR14 Ly $\alpha \mathrm{BAO}$ analysis performed in $[65,66]$. Note that, although the tension is lower for the eBOSS sample, which has roughly $20 \%$ more Lyman- $\alpha$ sources than BOSS, it does not necessarily mean that the tension will further decrease with a larger sample. The source of the tension, which could be new physics or a yet unknown systematic error, remains unknown, and forthcoming experiments, such as DESI, will help to clarify the issue.
} 
matrix and, for each realization, evaluate $w_{\mathrm{DE}}^{\mathrm{eff}}(a)$ from the conservation of the effective DE fluid. Averaging over the ensemble gives the mean and the uncertainty in $w_{\mathrm{DE}}^{\text {eff }}(a)$ shown in the plots. If a sampled $X(a)$ happens to have a $|X(a)|<10^{-5}$, we replace it with $X(a)=10^{-5}$ to prevent a singularity in $w_{\mathrm{DE}}^{\text {eff }}$. As expected, the uncertainty in $w_{\mathrm{DE}}^{\text {eff }}$ is very large at high redshifts in the case of the standard prior (left panel). This is because $w_{\mathrm{DE}}^{\text {eff }}(a)$ is determined by the derivative of $X(a)$ and each sampled $X(a)$ can fluctuate within the range allowed by the variance. In the case of the evidence-weighted reconstruction (right panel), $X(a)$ is a linear superposition of many reconstructions obtained with different priors. The different priors all prefer $X(a)$ to be constant, without enforcing any particular value of the constant. Thus, while there is $\sim 15 \%$ uncertainty around the value of $X(a)$ at high $z$, its derivative is zero with a much higher certainty, which explains why the uncertainty in $w_{\mathrm{DE}}^{\text {eff }}(a)$ is so small near $a=0$.

Interestingly, as shown in [28], the Bayesian evidence $(\Delta \ln E$ ) for the oscillatory features is positive at $2.8 \sigma$, and they appear equally prominently in both reconstructions in Fig. 1. We also note that, although the Bayesian evidence for dynamical DE is weak, it has increased over the years, with the dynamical pattern being largely consistent with the reconstruction performed in 2012 [67].

As we will see later, the oscillatory features in the reconstructions can, in certain circumstances, trigger fastgrowing instabilities in cosmological perturbations. Also, the oscillatory pattern and the overall increase in DE density are driven by entirely different datasets. For this reason, we have also considered $X(a)$ obtained by fitting a monotonic function to the reconstructions, which capture the overall increase but do not allow for oscillations. We take the form to be

$$
X_{\mathrm{fit}}(a)=A \tanh [B(a-C)]+D
$$

where the parameter $D$ is chosen such that $X_{\text {fit }}(a=1)=1$. The fitted functions and the corresponding DE EOS are shown with black solid and dashed lines, respectively, in Fig. 1. Thus, in what follows, we will consider four $X(a)$ histories:

H1.- - using the standard prior (green line, Fig. 1);

H1F. - the monotonic fit to H1 (black solid line);

H2. - evidence-weighted reconstruction (blue line);

H2F.- the monotonic fit to H2 (black dashed line).

As an increasing effective DE density cannot be realized in simple quintessence models, one is prompted to consider more complex gravity theories. In the next section we explore the GBD theories as a possible framework for explaining the observed DE dynamics.

\section{GENERALIZED BRANS-DICKE THEORIES AND WAYS TO RECONSTRUCT THEM}

The nonminimal coupling of the scalar field in the GBD theories could explain the observed "ghostly" behavior of the effective DE density. We stress that, in this context, the phantom dynamics is only an apparent phenomenon perceived by a cosmologist fitting the conventional Friedmann equation to data while being unaware of the nonminimal coupling.

The GBD action can be written as [68-70]

$$
\begin{aligned}
S= & \int d^{4} x \sqrt{-g}\left[\frac{m_{0}^{2}}{2} F(\phi) R-\frac{1}{2} K(\phi)(\partial \phi)^{2}-U(\phi)\right] \\
& +S_{m}\left[g_{\mu \nu}, \chi_{i}\right],
\end{aligned}
$$

where $m_{0} \equiv(8 \pi G)^{-1 / 2}$ is the Planck mass in terms of the Newton's constant $G$ measured on Earth, $\phi$ is the extra scalar degree of freedom (d.o.f.), $(\partial \phi)^{2} \equiv g^{\mu \nu} \partial_{\mu} \partial_{\nu} \phi, U(\phi)$ is the GBD potential and $S_{m}$ denotes the action for the matter fields $\chi_{i}$ minimally coupled to the (Jordan frame) metric $g_{\mu \nu}$. We set $K(\phi)=1$, as one can always do so by a redefinition of $\phi$. The modified Einstein equations are obtained by varying the action with respect to the metric $g_{\mu \nu}$ :

$$
F G_{\mu \nu}=\frac{1}{m_{0}^{2}}\left(T_{\mu \nu}^{\mathrm{m}}+T_{\mu \nu}^{\phi}\right)+\nabla_{\mu} \nabla_{\nu} F-g_{\mu \nu} \square F,
$$

where $\nabla_{\mu}$ denotes the covariant derivative with respect to the coordinate $x^{\mu}, \square \equiv g^{\mu \nu} \nabla_{\mu} \nabla_{\nu}, T_{\mu \nu}^{\mathrm{m}}$ is the matter energymomentum tensor and

$$
T_{\mu \nu}^{\phi} \equiv \partial_{\mu} \phi \partial_{\nu} \phi-g_{\mu \nu}\left[\frac{1}{2} \partial_{\alpha} \phi \partial^{\alpha} \phi+U(\phi)\right] .
$$

The equation of motion for the scalar field $\phi$ is then obtained by extremizing the action (4) with respect to variations of the field $\phi$ :

$$
\square \phi=U_{\phi}-\frac{m_{0}^{2}}{2} F_{\phi} R,
$$

where the subscript $\phi$ denotes a derivative with respect to $\phi$. For convenience we redefine the field, $\phi \rightarrow \phi / m_{0}$, to make it dimensionless, and the potential, $U \rightarrow U / m_{0}^{2}$, with the latter measured in $\mathrm{Mpc}^{-2}$ in agreement with the units convention in CAMB [71].

The freedom in choosing the two functions $F(\phi)$ and $U(\phi)$ translates into the ability of GBD theories to reproduce any expansion history. In particular, the effective $\mathrm{DE}$ density $X(a)$ defined in Eq. (1) can increase, and the effective DE EOS can cross -1 . To see this, we rewrite the modified Einstein equation (5) as 


$$
\begin{aligned}
G_{\mu \nu} & =\frac{1}{m_{0}^{2} F}\left\{T_{\mu \nu}^{m}+T_{\mu \nu}^{\phi}+\nabla_{\mu} \nabla_{\nu} F-g_{\mu \nu} \square F\right\} \\
& =\frac{1}{m_{0}^{2}}\left\{T_{\mu \nu}^{M}+\left(T_{\mathrm{DE}}^{\mathrm{eff}}\right)_{\mu \nu}\right\},
\end{aligned}
$$

where, in the second line, we have defined the effective DE stress-energy by absorbing into it all the terms on the righthand side other than the usual matter term, i.e.,

$$
\left(T_{\mathrm{DE}}^{\mathrm{eff}}\right)_{\mu \nu} \equiv F^{-1}\left\{T_{\mu \nu}^{\phi}+\nabla_{\mu} \nabla_{\nu} F-g_{\mu \nu} \square F+(1-F) T_{\mu \nu}^{m}\right\} .
$$

In a flat Friedmann-Robertson-Walker universe, the effective DE density is

$\rho_{\mathrm{DE}}^{\mathrm{eff}}=F^{-1}\left\{\dot{\phi}^{2} /\left(2 a^{2}\right)+U(\phi)-3 \mathcal{H} \dot{F} / a^{2}+(1-F) \rho_{m}\right\}$,

with the dot standing for a derivative with respect to the conformal time, while the effective DE pressure is

$p_{\mathrm{DE}}^{\mathrm{eff}}=F^{-1}\left\{\dot{\phi}^{2} /\left(2 a^{2}\right)-U(\phi)+\mathcal{H} \dot{F} / a^{2}+\ddot{F} / a^{2}\right\}$.

The $\mu=\nu=0$ component of Eq. (8) gives the Friedmann equation

$$
\mathcal{H}^{2}=\left(\frac{\dot{a}}{a}\right)^{2}=\frac{a^{2}}{3 m_{0}^{2}}\left[\rho_{m}(a)+\rho_{\mathrm{DE}}^{\mathrm{eff}}(a)\right],
$$

which can be recast in the form of Eq. (1).

Note that, by construction, the effective DE "fluid" is conserved, but its EOS,

$$
w_{\mathrm{DE}}^{\mathrm{eff}} \equiv \frac{p_{\mathrm{DE}}^{\mathrm{eff}}}{\rho_{\mathrm{DE}}^{\mathrm{eff}}}=\frac{\dot{\phi}^{2} /\left(2 a^{2}\right)-U(\phi)+\mathcal{H} \dot{F} / a^{2}+\ddot{F} / a^{2}}{\dot{\phi}^{2} /\left(2 a^{2}\right)+U(\phi)-3 \mathcal{H} \dot{F} / a^{2}+(1-F) \rho_{M}},
$$

is not always well defined because $\rho_{\mathrm{DE}}^{\text {eff }}$ in the denominator is allowed to change sign due to the new terms generated by the nonminimal coupling $F(\phi)$. Thus, as previously noted in $[36,72]$, observing $w_{\mathrm{DE}}^{\text {eff }}<-1$, or finding that $\rho_{\mathrm{DE}}^{\text {eff }}$ changes its sign, could be a smoking gun of interactions in the dark sector, modified gravity or extra dimensions (e.g., phantom brane models of dark energy [73,74]).

The idea of reconstructing the GBD Lagrangian from a given expansion history was previously explored in [75-77], motivated by the fact that the Hubble function $H(a)$ inferred from the supernovae data available at that time showed a preference for an effective phantom DE equation of state, $w_{\mathrm{DE}}^{\mathrm{eff}}<-1$. As they have shown, one can, in principle, reconstruct both functions $F(\phi)$ and $U(\phi)$ if, in addition to $H(a)$, one knows the evolution of the growth of the matter density contrast $\delta(a)$. Another interesting example is the $f(R)$ gravity where the only unknown function is the function $f$ itself and the full reconstruction can be done with the sole knowledge of the expansion history $H(a)$ [45,78].
In the present work we adopt a slightly different approach. Since the growth of perturbations is rather complicated to extract in a model-independent way because of the redshift-space distortions, nonlinearities, bias, etc., we attempt to reconstruct only one of the functions, namely $U(\phi)$, while the other is chosen to either have a given functional form $F(\phi)$ (model 1) or a given parameterized time dependence $F(a)$ (model 2). We will analyze these two cases separately.

While exploring the parameter space, which includes the initial conditions for the scalar field, we restrict to solutions in which the net change in $F(\phi)$ is under $10 \%$, to satisfy the big bang nucleosynthesis $(\mathrm{BBN})$ constraints on the variation of the Newton's constant. There are also stringent constraints on the value of $F(\phi)$ today coming from laboratory and Solar System tests [79], although interpretation of these constraints can be modified in theories in which the force mediated by the scalar field is screened, as in "chameleon" [80] or "symmetron" [81] models. Since our aim is to explore the ability of cosmological probes to rule out scalar-tensor theories independently from the latter, we do not take these constraints into account. Finally, we also check for various types of instabilities using the procedure implemented in EFTCAMB [82,83]. Specifically, we check for ghost, gradient and mass instabilities discussed in detail in [84] and briefly reviewed below.

After expanding the action up to the second order in perturbations of the metric and matter fields, and removing spurious d.o.f., one can isolate the action for the propagating scalar and tensor d.o.f. [85]. The conditions for avoiding instabilities can then be formulated in Fourier space in terms of the corresponding kinetic, gradient and mass matrices as follows:

(1) No-ghost.-A ghost instability develops when the kinetic term of a field is negative. In the presence of multiple propagating d.o.f., a positive definite kinetic matrix guarantees that no ghosts will develop. In practice, this requirement needs to be imposed only at high energies, i.e., in the high- $k$ limit, since an infrared ghost does not lead to catastrophic instabilities [86].

(2) No-gradient.-Gradient instabilities arise in the high- $k$ regime when the speed of propagation is imaginary. The sound speeds of the propagating d.o.f. can be identified from the dispersion relations that result from the quadratic action after diagonalizing the kinetic matrix. In order to avoid gradient instabilities we impose $c_{s}^{2}>0$ for all the d.o.f.

(3) No-tachyon.-Whenever the mass matrix of the Hamiltonian contains a negative eigenvalue, the mass instability plagues the low- $k$ regime with the development of a tachyon [85]. The rate of the instability needs to be taken into account. We will assume that the GBD Hamiltonian exhibits a tachyonic instability when at low momenta a mass 
eigenvalue $\mu_{i}$ becomes negative and evolves rapidly, i.e., $\left|\mu_{i}\right| \gg H^{2}$. Thus, for a theory to be viable, we require $\mu_{i}>0$ or, alternatively, $\left|\mu_{i}\right| \lesssim H^{2}$.

This set of conditions was shown to guarantee stability over the full range of linear scales [84] and was implemented in a private version of EFTCAMB.

Public versions of EFTCAMB, as well as other EinsteinBoltzmann solvers like HiClass [87], do not contain the mass condition. Instead, in addition to checking for the no-ghost and no-gradient instabilities, they impose a set of mathematical conditions that prevent the development of exponentially diverging solutions. The latter are worked out from the linear order equation for the scalar field perturbation and are meant to protect against the mass instabilities as well as the ghost and gradient instabilities that could have possibly evaded the checks based on some approximations necessary in setting the conditions. When a mathematical condition is violated, one cannot easily tell which of the three types of instabilities was responsible. In our analysis we used both methods. Namely, we checked for the ghost, gradient and mass stability conditions, as well as using the publicly available stability check that combines the ghost, the gradient and the mathematical conditions.

\section{A. Model 1: Reconstructing GBD for a given $F(\phi)$}

Given the functional form of $F(\phi)$, we can reconstruct $U(\phi)$ from a given expansion history. We take

$$
F(\phi)=\exp (\xi \phi),
$$

which is a form motivated by high-energy theories, e.g., a nonminimally coupled dilaton field representing compactified extra dimensions with the dimensionless parameter $\xi$ controlling the coupling strength.

We begin by writing the two Friedmann equations as

$$
\begin{gathered}
\mathcal{H}^{2}=\frac{1}{\mathcal{D}} \frac{\rho a^{2}}{3 m_{0}^{2}}+\frac{1}{\mathcal{D}} \frac{U a^{2}}{3}, \\
\mathcal{G} \frac{\ddot{a}}{a}=2 \mathcal{D} \mathcal{H}^{2}-\frac{a^{2} \mathcal{H}^{2}}{2}\left(\frac{1}{3}+F_{\phi \phi}\right)\left(\phi^{\prime}\right)^{2} \\
-\frac{1}{2} \frac{(\rho+P) a^{2}}{m_{0}^{2}}-\frac{1}{2} F_{\phi} \mathcal{H}^{2}\left(\phi^{\prime \prime}-\phi^{\prime}\right),
\end{gathered}
$$

where

$$
\begin{gathered}
\mathcal{D}=F-\frac{1}{6}\left(\phi^{\prime}\right)^{2}+F_{\phi} \phi^{\prime}, \\
\mathcal{G}=F+\frac{1}{2} F_{\phi} \phi^{\prime},
\end{gathered}
$$

and the prime denotes derivatives with respect to $N \equiv \ln a$. Equation (15) can be rewritten as an equation for the background evolution of $\phi$ :

$$
\begin{aligned}
\phi^{\prime \prime}= & -\frac{1+F_{\phi \phi}}{F_{\phi}}\left(\phi^{\prime}\right)^{2}+\left(1+\frac{13 E_{m}+4 E_{r}-E_{\nu}^{\prime}-\Omega_{\Lambda} X^{\prime}}{E_{m}+E_{r}+E_{\nu}+\Omega_{\Lambda} X}\right) \phi^{\prime} \\
& +\frac{1}{F_{\phi}} \frac{(F-1)\left(3 E_{m}+4 E_{r}-E_{\nu}^{\prime}\right)-F \Omega_{\Lambda} X^{\prime}}{E_{m}+E_{r}+E_{\nu}+\Omega_{\Lambda} X},
\end{aligned}
$$

where $E_{m} \equiv \rho_{m} / \rho_{\text {crit }}^{0}$ includes $\mathrm{CDM}$ and baryons, $E_{r} \equiv \rho_{r} / \rho_{\text {crit }}^{0}$ includes photons and massless neutrinos and $E_{\nu} \equiv \rho_{\nu} / \rho_{\text {crit }}^{0}$ includes massive neutrinos species only. Equation (18) can be solved given the functional form (13) of $F(\phi)$ and the DE density evolution $X(a)$. Given the solution $\phi(a)$, one can find the potential $U(a)$ from Eq. (14), namely,

$$
\begin{aligned}
U a^{2}= & 3 \mathcal{D} H_{0}^{2} a^{2}\left(E_{m}+E_{r}+E_{\nu}+\Omega_{\Lambda} X\right) \\
& -3 H_{0}^{2} a^{2}\left(E_{m}+E_{r}+E_{\nu}\right) .
\end{aligned}
$$

If $\phi(a)$ is monotonic, it can be inverted to obtain $a(\phi)$ and, thus, $U(\phi)$ for the range of $\phi$ covered by the evolution.

Solving Eq. (18) requires setting the value of the field $\phi_{\text {ini }}$ and its derivative $\phi_{\text {ini }}^{\prime}$ at some initial time $a_{\text {ini. }}$. To preserve the success of $\Lambda \mathrm{CDM}$ in explaining the $\mathrm{BBN}$ and the peak structure of the CMB spectrum, we assume that gravity was close to GR at early times, so that $F(\phi)=1$ for $a \leq a_{\text {ini }}$, but could start deviating from unity at later times. For $F(\phi)=\exp (\xi \phi)$ this means $\phi_{\text {ini }}=0$ and, to explain the features in the reconstructed DE density discussed in the previous section, we will need $a_{\text {ini }} \lesssim 0.1$. Thus, in addition to providing $X(a)$, we have to specify three parameters: $\xi, a_{\text {ini }}$ and $\phi_{\text {ini }}^{\prime}$.

For the H1, H1F and $\mathrm{H} 2$ background histories, reconstructed model 1 theories contain fast-growing mode instabilities for all choices of initial conditions. For the perturbations around these backgrounds we find that both the mass and the mathematical conditions are not satisfied. It appears that the large rapid increases in $X(a)$ present in $\mathrm{H} 1, \mathrm{H} 1 \mathrm{~F}$ and $\mathrm{H} 2$ drive the solution towards instability, which could, in principle, be prevented by an appropriate choice of $F(\phi)$. However, the model 1 coupling function $F(\phi)=\exp (\xi \phi)$ is monotonic and is unable to prevent the onset of instability.

In the case of $\mathrm{H} 2 \mathrm{~F}$, which is monotonic and with a relatively small change in $X(a)$, we are able to find viable solutions despite finding negative mass eigenvalues. There, the tachyonic instability corresponding to the negative mass eigenvalues develops on timescales comparable to the Hubble rate allowing for growth of cosmic structure that is in reasonable agreement with observations.

To summarize, we find that model 1 reconstructions from all four expansion histories are plagued by a mass instabilities. For H1, H1F and H2, this instability develops on timescales small enough for the mathematical condition to detect diverging solutions. In the case of $\mathrm{H} 2 \mathrm{~F}$ the characteristic timescale is longer and, while our approximate bound 


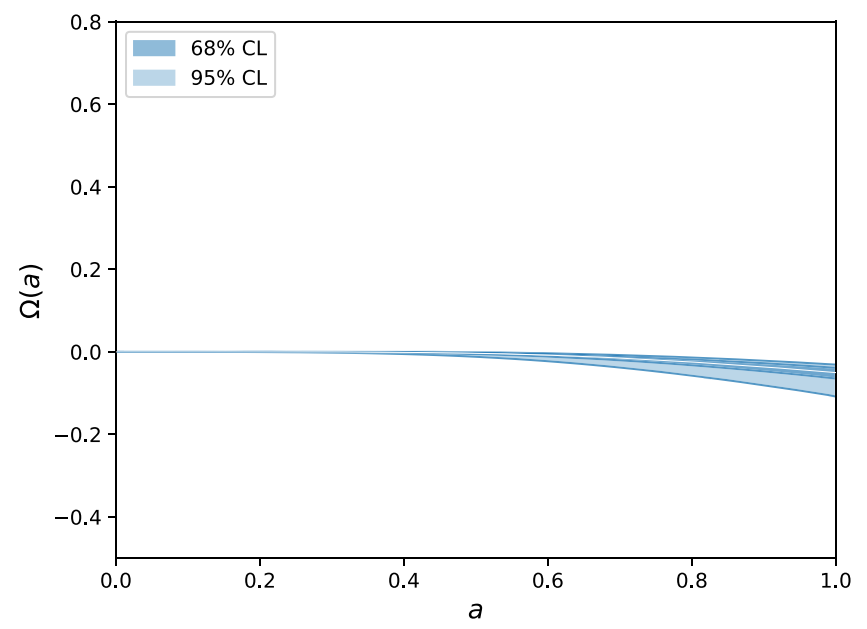

FIG. 2. The viable range of values of the nonminimal coupling $\Omega(a) \equiv F(a)-1$ for the model 1 GBD theories reconstructed from the $\mathrm{H} 2 \mathrm{~F}$ expansion history. The confidence level (CL) regions are obtained by sampling parameters $\phi_{\text {ini }}^{\prime}, \xi$ and $\log _{10} a_{\text {ini }}$ as described in the text.

of $\left|\mu_{i}\right|<H^{2}$ is not satisfied, the instability does not develop to the point of giving diverging solutions.

Figure 2 shows the allowed range of the nonminimal coupling function $\Omega(a) \equiv F(\phi(a))-1$ for GBD theories reconstructed from $\mathrm{H} 2 \mathrm{~F}$. It is obtained by uniformly sampling parameters $\left(\phi_{\mathrm{ini}}^{\prime}, \log _{10} a_{\mathrm{ini}}, \xi\right)$ from the intervals

$\frac{\phi_{\text {ini }}^{\prime}}{m_{0}} \in\left[-10^{-6}, 10^{-6}\right], \quad \log _{10} a_{\text {ini }} \in[-3,-1], \quad \xi \in[0.1,10]$,

solving for the evolution of $\phi$ and selecting solutions that have $\Omega$ within the allowed range and satisfy the stability condition. The shaded regions in Fig. 2 indicate the confidence level (CL) for having a particular value of $\Omega$ at a given $a$, while the dark line in the middle shows the mean. Examining the numerical solutions, we find that, as expected, the increasing effective DE density drives the field to negative values, resulting in $F(\phi)<1$ and a larger $G_{\text {eff }} \propto G / F(\phi)$.

For illustration, in Fig. 3 we show the potential $U(\phi)$ for four GBD theories reconstructed from the H2F DE density with $\phi_{\text {ini }}^{\prime} / m_{0}=0$ and $a_{\text {ini }}=10^{-2}$ and $\xi=0.5,1.5,3$ and 10 , respectively. We can see how in all four cases the potential has a cusp at the origin. Stronger couplings lead to steeper potentials. Their shapes resemble the potential in chameleonlike models [88,89], $V(\phi) \propto|\phi|^{-n}$, although the dynamics here is completely different. In the chameleon model, the field tracks the minimum of the effective potential, with the coupling function $F(\phi)$ slowly increasing with the evolution. In our reconstructed theories, the field $\phi$ starts at the top of the cusp and rolls down the potential, with $F(\phi)$ decreasing as it rolls.

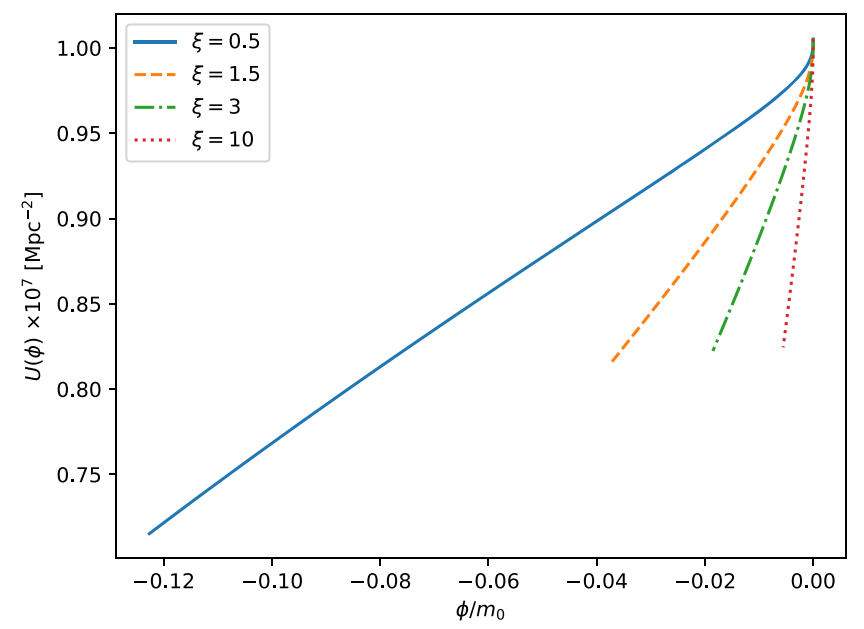

FIG. 3. The potential $U(\phi)$ in four representative model 1 GBD theories reconstructed from the $\mathrm{H} 2 \mathrm{~F}$ expansion history with the same $\phi_{\text {ini }}^{\prime}$ and $\log _{10} a_{\text {ini }}$ and our different values of the coupling parameter $\xi$.

\section{B. Model 2: Reconstructing GBD with a parameterized $\boldsymbol{F}(\boldsymbol{a})$}

We now change the approach and, instead of working with a given $F(\phi)$, we directly specify the time dependence of $F$, i.e., $F(a)$. A similar approach was used in $[76,77]$ to reconstruct the GBD Lagrangian from the expansion history inferred from an early SNe Ia dataset. In this case, we start by writing the modified Friedmann equations as

$$
\begin{aligned}
\mathcal{H}^{2}= & \frac{1}{3 m_{0}^{2}} \frac{1}{F+a F^{\prime}}\left[\rho a^{2}+\frac{1}{2} \dot{\phi}^{2}+U a^{2}\right], \\
\dot{\mathcal{H}}= & \frac{1}{F+\frac{1}{2} a F^{\prime}}\left\{\left[F+2 a F^{\prime}+a^{2} F^{\prime \prime}\right] \mathcal{H}^{2}\right. \\
& \left.-\frac{1}{2 m_{0}^{2}}\left[P a^{2}+\frac{1}{2} \dot{\phi}^{2}-U a^{2}\right]\right\},
\end{aligned}
$$

where primes denote derivatives with respect to the scale factor and overdots denote derivatives with respect to the conformal time $\tau$. We can then use (21) to eliminate the potential $U$ in (22) to write

$$
\begin{aligned}
\dot{\mathcal{H}}= & \left\{\left[\frac{5}{2} F+\frac{7}{2} a F^{\prime}+a^{2} F^{\prime \prime}\right] \mathcal{H}^{2}-\frac{(\rho+P) a^{2}}{2 m_{0}^{2}}-\frac{1}{2 m_{0}^{2}} \dot{\phi}^{2}\right\} \\
& \times\left(F+\frac{1}{2} a F^{\prime}\right)^{-1} .
\end{aligned}
$$

One can then solve the above equation for $\dot{\phi}$ and use it in (21) to obtain a solution for $U(a)$ : 

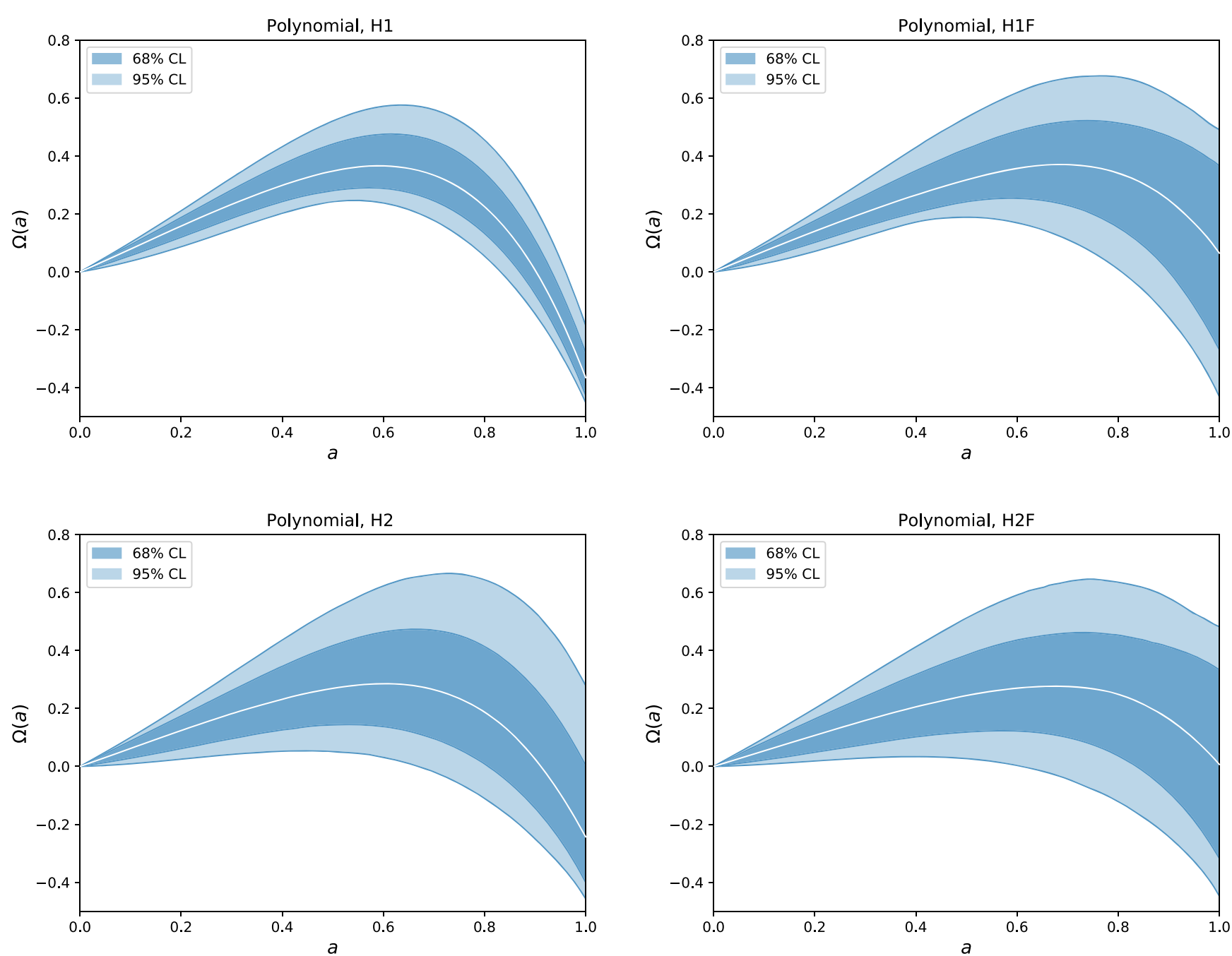

FIG. 4. The distribution of nonminimal coupling functions $\Omega(a)=F(a)-1$ obtained using the model 2 polynomial parametric form for stable GBD theories with the four effective DE histories H1, H1F, H2 and H2F.

$$
\begin{aligned}
\frac{U a^{2}}{m_{0}^{2}}= & \mathcal{H}^{2}\left[\frac{1}{2} F-\frac{1}{2} a F^{\prime}-a^{2} F^{\prime \prime}\right] \\
& +\frac{(P-\rho) a^{2}}{2 m_{0}^{2}}+\dot{\mathcal{H}}\left[F+\frac{1}{2} a F^{\prime}\right] .
\end{aligned}
$$

With the known $U(a)$, one can solve for the kinetic energy $\dot{\phi}^{2}$ from (21) and complete the solution by solving the differential equation to find $\phi(a)$. With the field $\phi(a)$ known, one can convert $U(a)$ and $F(a)$ into $U(\phi)$ and $F(\phi)$, thus reconstructing the functional form of the theory for the range of $\phi(a)$ covered by the solution.

To explore a broad range of possible $F(a)$ histories we adopt a polynomial parametric form

$$
F(a)=1+\sum_{i=1}^{5} \alpha_{i} a^{i},
$$

with coefficients $\alpha_{i}$ sampled uniformly from

$$
\alpha_{i} \in[-1,1]
$$

This range is chosen to favor positive values of $F(a)$ close to unity as required by existing bounds.

With $H(a)$ and $F(a)$ specified, one can use EFTCAMB [90], as described in the next section, to compute the cosmological observables.

We have generated samples of $F(a)$ using the parameterized form (25) and performed reconstructions of the GBD theories for each of the four $X(a)$ histories shown in Fig. 1. The viable ranges of $F(a)$ functions in each case are shown in Fig. 4. One can see that for H1, H1F and H2F, in which $X(a)$ has a large increase or is nonmonotonic, $F(a)$ must be nonmonotonic to avoid instabilities. In the case of $\mathrm{H} 2 \mathrm{~F}$, which has a gently increasing monotonic $X(a)$, a monotonic $F(a)$ has a small probability but is not excluded.

To reconstruct $U(\phi)$ and $F(\phi)$ one needs to solve a firstorder ordinary differential equation for $\phi$, which requires specifying a boundary condition, such as the value of the 

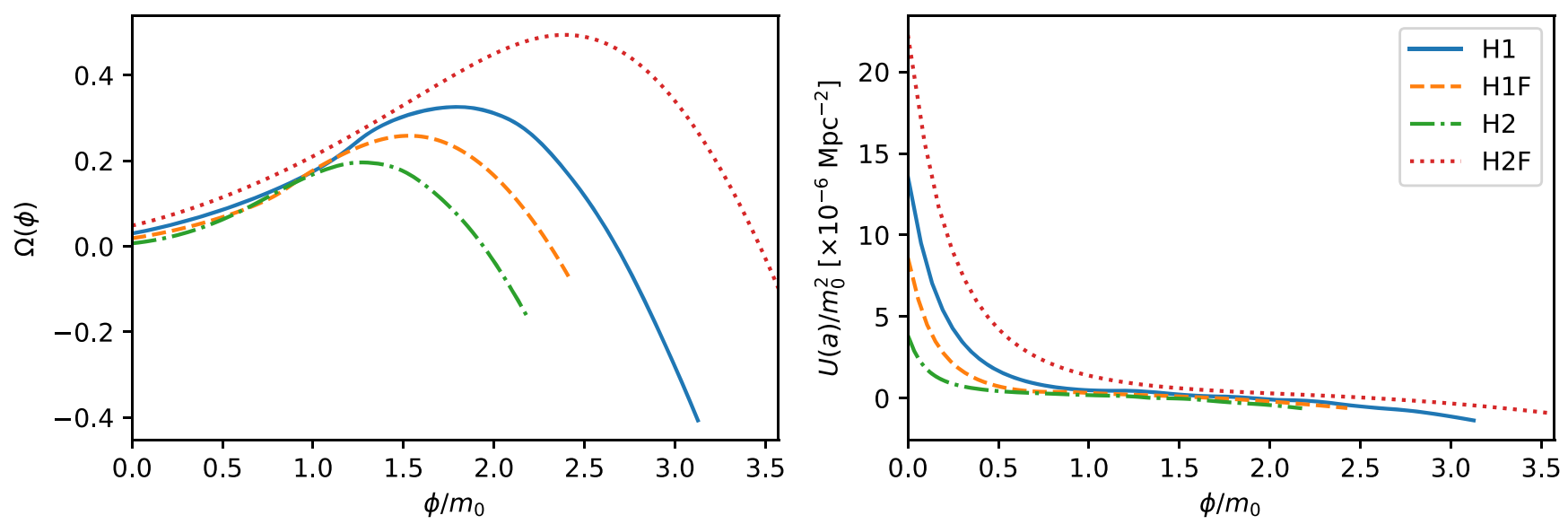

FIG. 5. The coupling function $\Omega(\phi)=F(\phi)-1$ and the potential $U(\phi)$ for four representative model 2 theories reconstructed from the $\mathrm{H} 1, \mathrm{H} 1 \mathrm{~F}, \mathrm{H} 2$ and $\mathrm{H} 2 \mathrm{~F}$ expansion histories.

field at an initial time $a_{\text {ini }}$. This means we can only reconstruct $U(\phi)$ and $F(\phi)$ up to an arbitrary shift in the value of $\phi$. The shift has no physical significance, as all the observables are already fully determined. Hence, without loss of generality, we take $a_{\text {ini }}=0.001$ and $\phi\left(a_{\text {ini }}\right)=0$.

Figure 5 shows the nonminimal coupling function $\Omega(\phi)=F(\phi)-1$ and the potential $U(\phi)$ for four representative theories reconstructed from $\mathrm{H} 1, \mathrm{H} 1 \mathrm{~F}, \mathrm{H} 2$ and HF2. We see that $\Omega(\phi)$ is nonmonotonic in these representative cases. The potentials have a runaway shape, being seemingly unbounded from below for large values of the field, although one should keep in mind that the shape is only known over the range covered by the evolution of the field. One can also see small bumps in the potentials derived from $\mathrm{H} 1$ and $\mathrm{H} 2$, needed to accommodate oscillations in $X(a)$.

\section{COSMOLOGICAL OBSERVABLES IN RECONSTRUCTED GBD THEORIES}

Next we investigate the cosmological implications of the reconstructed GBD theories by computing the CMB anisotropy and the matter power spectra, along with the cross-correlation of the CMB temperature and Galaxy number counts (GNC), which probes the ISW effect. It is relatively straightforward to calculate these observables using EFTCAMB [82,83,91], which is an implementation of the effective field theory of dark energy (EFTofDE) [92,93] in the popular Boltzmann solver САMB [71]. In the EFTofDE approach, the most general action for the cosmological background and perturbations in scalartensor theories can be written in the unitary gauge, in which the scalar field is uniform on hypersurfaces of constant time, as an expansion in increasing rank-ordered operators invariant under spatial diffeomorphisms. The time-dependent expansion coefficients are referred to as the EFT functions. The part of the EFT action of relevance to the GBD theories is
$S=\int d^{4} x \sqrt{-g}\left\{\frac{m_{0}^{2}}{2}[1+\Omega(\tau)] R+\Lambda(\tau)+c(\tau) a^{2} \delta g^{00}\right\}$,

where $\tau$ is the conformal time, $\delta g^{00}=g^{00}+1$ is the metric tensor perturbation, and $\Omega, \Lambda$ and $c$ are the EFT functions. The GBD theories reconstructed in the previous section can be mapped onto the EFT formalism via

$$
\begin{gathered}
\Omega(a)=F(\phi(a))-1, \\
\frac{c a^{2}}{m_{0}^{2}}=\frac{1}{2} \mathcal{H}^{2}\left(\phi^{\prime}\right)^{2}, \\
\frac{\Lambda a^{2}}{m_{0}^{2}}=\frac{1}{2} \mathcal{H}^{2}\left(\phi^{\prime}\right)^{2}-U a^{2} .
\end{gathered}
$$

With this mapping we can use EFTCAMB to compute the CMB spectra and other cosmological observables.

As the initial time of the reconstruction $a_{\text {ini }}$ is after the epoch of recombination, the only differences in the CMB anisotropy spectrum compared to the $\Lambda$ CDM model can be due to the change in the expansion history, that modifies the distance to last scattering and shifts the positions of the peaks and troughs, and due to the different evolution of the gravitational potentials which affects the late-time ISW contribution to anisotropy. In the latter case, the phenomenology of GBD theories has three competing effects. First, just like in the case of $\Lambda \mathrm{CDM}$, the accelerating expansion causes a decay of the metric potentials. In our reconstructed expansion histories the matter-dominated era lasts longer, thus delaying the decay of the potentials. Second, the background value of the effective Newton's constant that determines the rate of gravitational clustering is $G / F(\phi)$ and can be larger or smaller than $G$, depending on the dynamics of the coupling function, correspondingly increasing or decreasing the rate at which the metric 

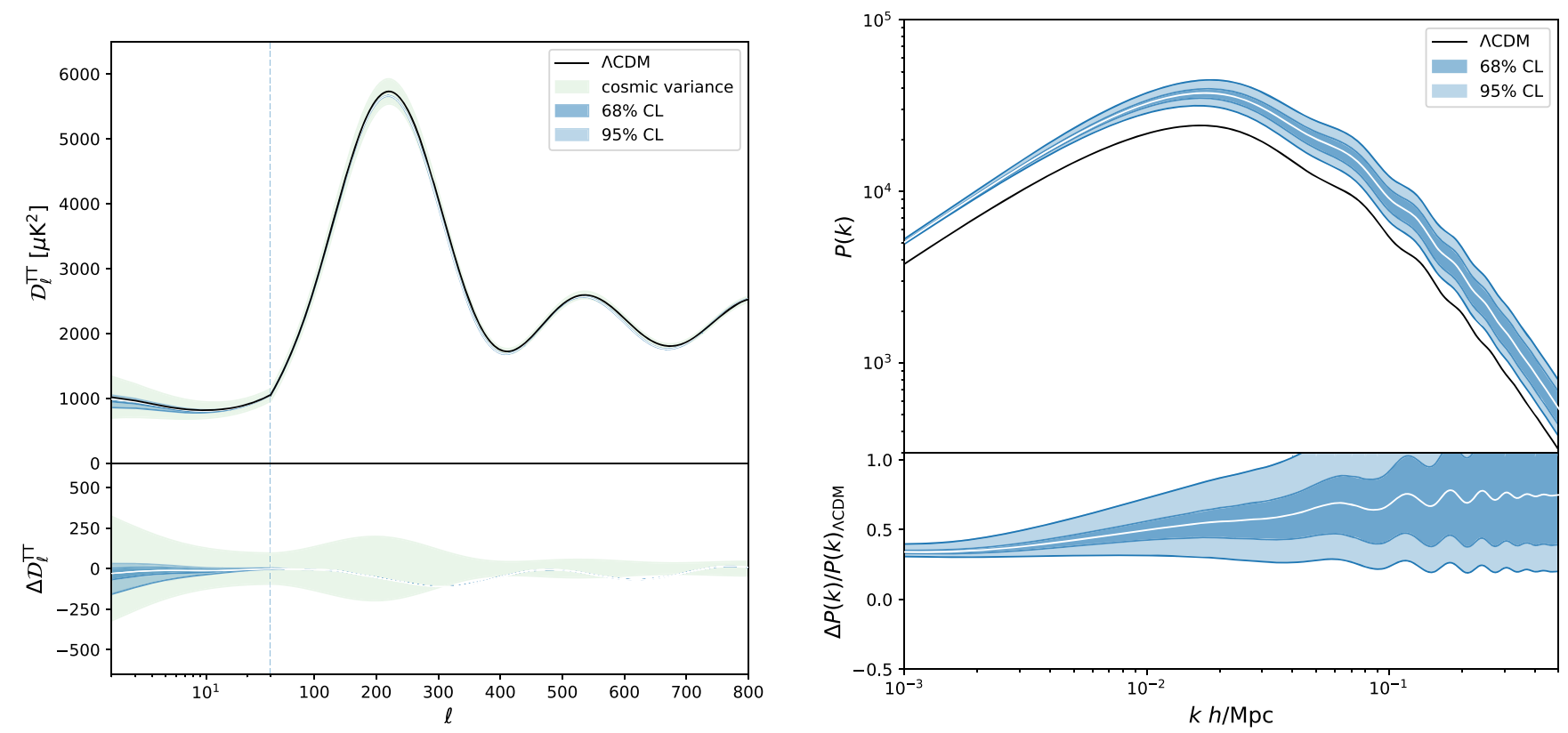

FIG. 6. Left panel: The distribution of CMB anisotropy spectra corresponding to stable model 1 theories reconstructed from the H2F $\mathrm{DE}$ density and the relative differences with respect to the $\Lambda \mathrm{CDM}$ best-fit model. The uncertainty due to cosmic variance around the $\Lambda C D M$ best fit is shown for reference. As expected the GBD theories affect mainly the ISW effect at low $\ell$. Right panels: The linear matter power spectrum (at redshift $z=0$ ) for model 1 theories reconstructed from $\mathrm{H} 2 \mathrm{~F}$ and the relative difference from the $\Lambda \mathrm{CDM}$ best fit.

potentials evolve. Third, the scalar field mediates a fifth force on scales smaller that the Compton wavelength of the field, which enhances the growth of the potentials. It is practically impossible to isolate these effects in the $\mathrm{CMB}$ anisotropy spectrum, since it only probes the square of the overall integral of the ISW signal. However, one can learn more by studying the correlation of CMB temperature with galaxy distribution at different redshifts $[39,40]$. In particular, a characteristic signature of the fifth force would be a negative galaxy-CMB correlation at high redshifts, where one normally expects no ISW signal. A change in the background value of the gravitational coupling could show as either a positive or negative signal, depending on its evolution.

The CMB temperature and GNC cross-correlation angular power spectrum can be written as

$$
C_{\ell}^{T g}=\frac{2}{\pi} \int d k k^{2} \Delta_{\ell}^{\mathrm{ISW}}\left(k, \tau_{0}\right) \Delta_{\ell}^{\mathrm{GNC}}\left(k, \tau_{0}\right) \mathcal{P}_{\mathcal{R}}(k)
$$

where the ISW transfer function is given by

$$
\Delta_{\ell}^{\mathrm{ISW}}\left(k, \tau_{0}\right)=-\int_{\tau^{*}}^{\tau_{0}} d \tau(\dot{\Phi}+\dot{\Psi}) j_{\ell}\left[k\left(\tau_{0}-\tau\right)\right]
$$

and the GNC transfer function is given by

$$
\begin{aligned}
\Delta_{\ell}^{\mathrm{GNC}}\left(k, \tau_{0}\right)= & \int_{0}^{\tau_{0}} d \eta W(z) \frac{d z}{d \tau} b_{g}(\tau, k) \delta(\tau, k) j_{\ell}\left[k\left(\tau_{0}-\tau\right)\right] \\
& + \text { corrections. }
\end{aligned}
$$

In the above, $\Phi$ and $\Psi$ are the Newtonian gauge metric potentials in Fourier space, $\delta(k, \tau)$ is the matter density contrast, $W(z)$ is the window function that selects galaxies in the given redshift range, and $b_{g}$ is the galaxy bias. The term "corrections" in Eq. (33) denotes collectively the redshiftspace-distortion corrections, lensing terms, and other contributions suppressed by $\mathcal{H} / k$ [94]. The cross-correlation spectra are then computed using the EFTCAMB patch for CAMB sources ${ }^{2}[94,96]$.

Since we are not interested in fitting the parameters of the GBD theories to data, but rather in investigating the qualitative features of the ISW effect, we choose to show the cross-correlation in three Gaussian windows $W_{1}, W_{2}$ and $W_{3}$ centered at redshifts $z_{1}=0.5, z_{2}=1$ and $z_{3}=3$. The widths of the window functions are $\sigma_{1}=0.05, \sigma_{2}=0.1$ and $\sigma_{3}=0.5$. The galaxy bias $b_{g}$ is, in general, time and scale dependent. On large scales, relevant for the cross-correlation with $\mathrm{CMB}$, one expects the scale dependence of the bias to be weak and the time dependence to have a simple polynomial dependence (see [97]). The bias is degenerate with

\footnotetext{
${ }^{2}$ The latest EFTCAMB patch is not yet compatible with the latest CAMB. In its last update, САMB and CAMB sources have been merged, so we used the last available iteration of CAMB sources at Ref. [95].
} 

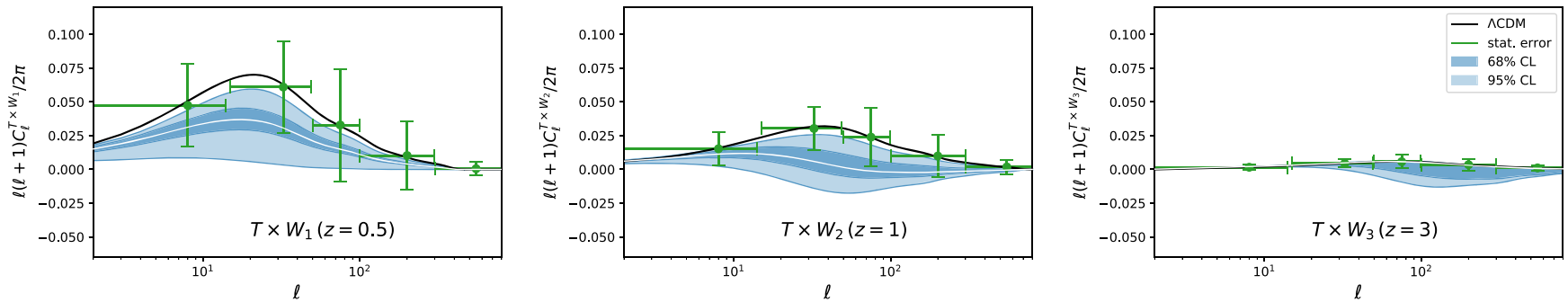

FIG. 7. The distribution of the CMB and Galaxy number counts cross-correlation spectra in three redshift bins at $z=0.5,1$ and 3 , corresponding to the viable model 1 theories reconstructed from H2F DE density. The best-fit $\Lambda$ CDM spectra are shown for reference. The green error bars show the uncertainty due to cosmic variance in the $\Lambda \mathrm{CDM}$ prediction in several wide bins of $\ell$. As one can see, the CMB temperature-GNC cross-correlations for the GBD theories can be either positive or negative.

the ISW amplitude, but one can calibrate it by jointly studying the GNC autocorrelations and the cross-correlations between GNC and galaxy lensing. As we are only interested in demonstrating the general features of the ISW signal, we fix the galaxy bias to $b_{g}=1$.

\section{A. Observables for model 1}

In the left panel of Fig. 6 we show the distribution of CMB temperature anisotropy spectrum $\mathcal{D}_{\ell} \equiv \ell(\ell+1)(2 \pi)^{-1} C_{\ell}$

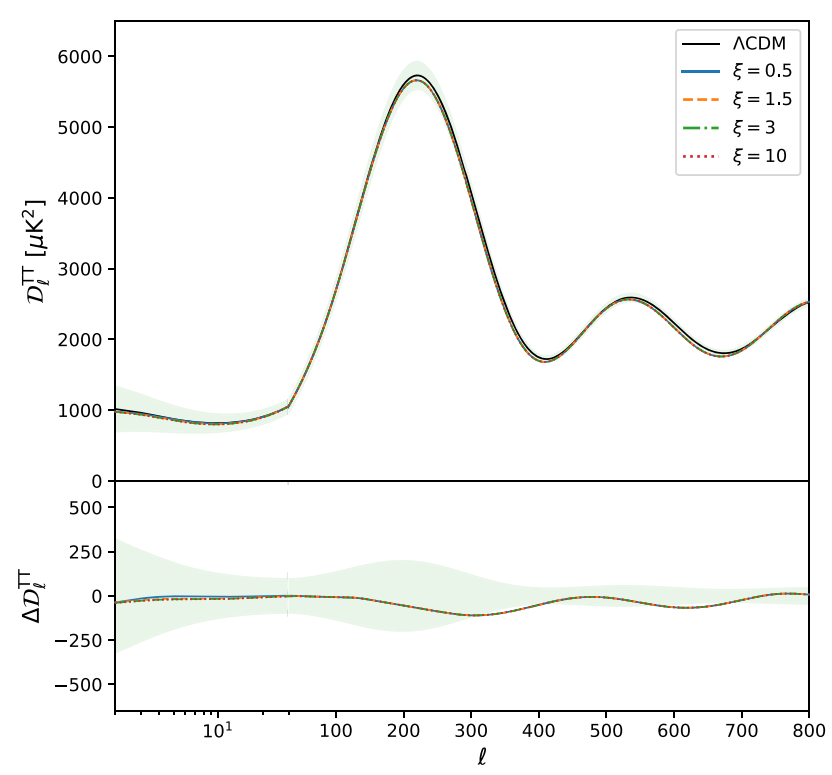

for model 1 theories reconstructed from the H2F DE density obtained by sampling the parameter space as described in Sec. III A. The shaded regions represent the CL regions to find $\mathcal{D}_{\ell}$ in the corresponding range, while the white lines show the mean values. In this sampling procedure we used the cosmological parameters obtained in the reconstruction of $X(a)$ in [28], except for the parameters setting the primordial power spectrum which were not constrained in [28], and for which we used the best-fit $\Lambda$ CDM values.

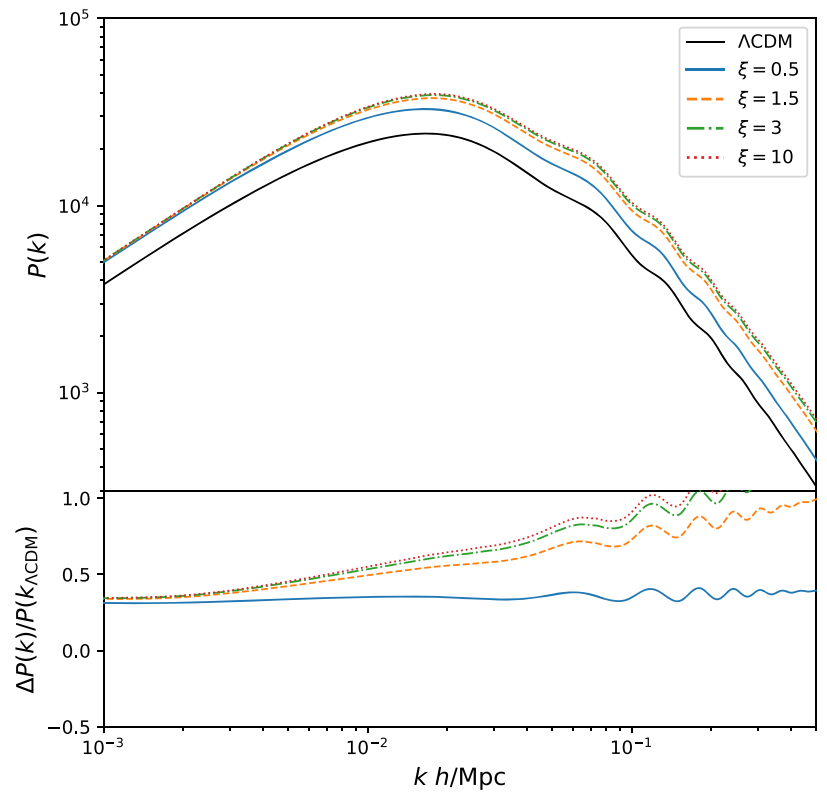

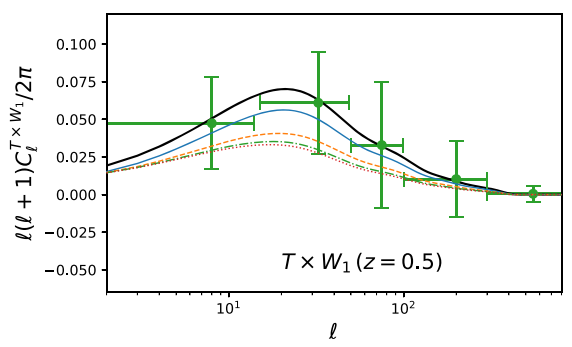
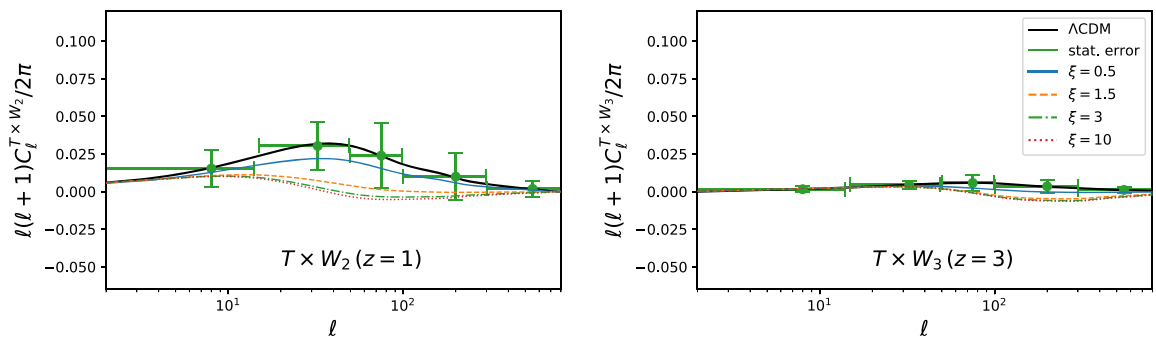

FIG. 8. Cosmological observables for four representative model 1 theories reconstructed from the H2F DE density. The cosmic variance uncertainty around the $\Lambda \mathrm{CDM}$ best fit is shown for reference. 
The light green band shows the irreducible statistical uncertainty in $\mathcal{D}_{\ell}$ due to cosmic variance based on the $\Lambda \mathrm{CDM}$ model. As the $\mathcal{D}$ measured by Planck are cosmic variance limited over most of the cosmologically relevant $\ell$ [98], the shown uncertainty is representative of current data.

As expected, we observe a modified ISW effect at small $\ell$. The small differences in the high- $\ell$ part of the spectra are mainly due to the different distance to the last scattering surface (because of the different expansion history) which causes a shift in the peaks and also because of the different baryon and CDM densities $\Omega_{b} h^{2}$ and $\Omega_{c} h^{2}$ in the $X(a)$ vs $\Lambda \mathrm{CDM}$ cases. These high- $\ell$ differences are well within the cosmic variance band and would likely be accommodated by adjusting other parameters in a comprehensive MonteCarlo Markov Chains parameter estimation.

The right panel of Fig. 6 shows the linear matter power spectrum. First of all, one can note an overall shift upwards for the GBD theories. At early times, before DE begins to dominate the background dynamics, the Planck best-fit $\Lambda \mathrm{CDM}$ model has more DE density than the GBD models with the reconstructed DE. This means that in the GBD models the matter-dominated era lasts slightly longer than in the $\Lambda \mathrm{CDM}$ model, allowing matter to cluster more, hence the overall shift upwards of the matter power
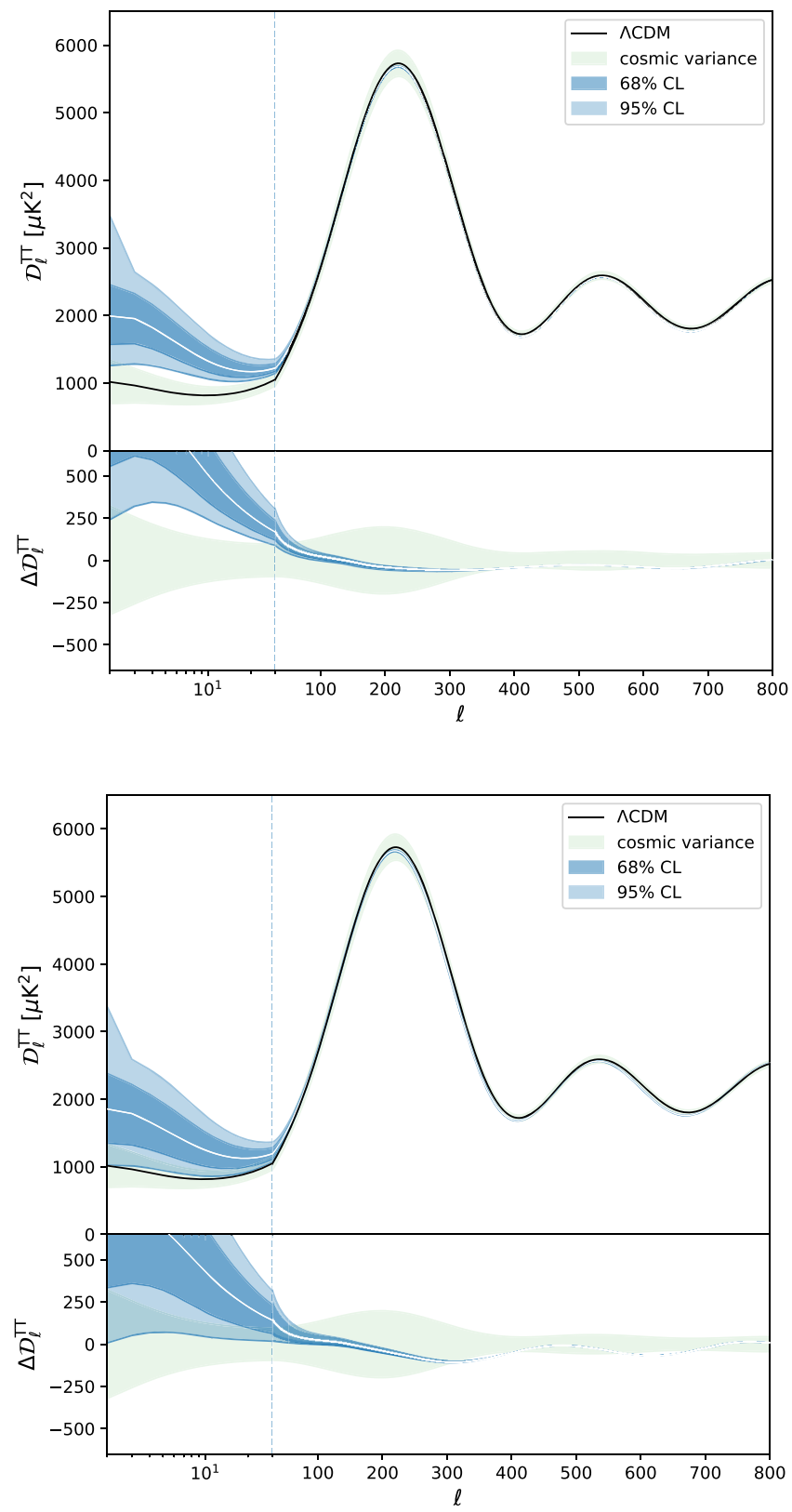

FIG. 9. The distribution of CMB temperature anisotropy spectra for model 2 theories reconstructed using the H1, H1F, H2 and H2F expansion history. The best-fit $\Lambda$ CDM spectra, along with the statistical error bars, are shown for reference. 


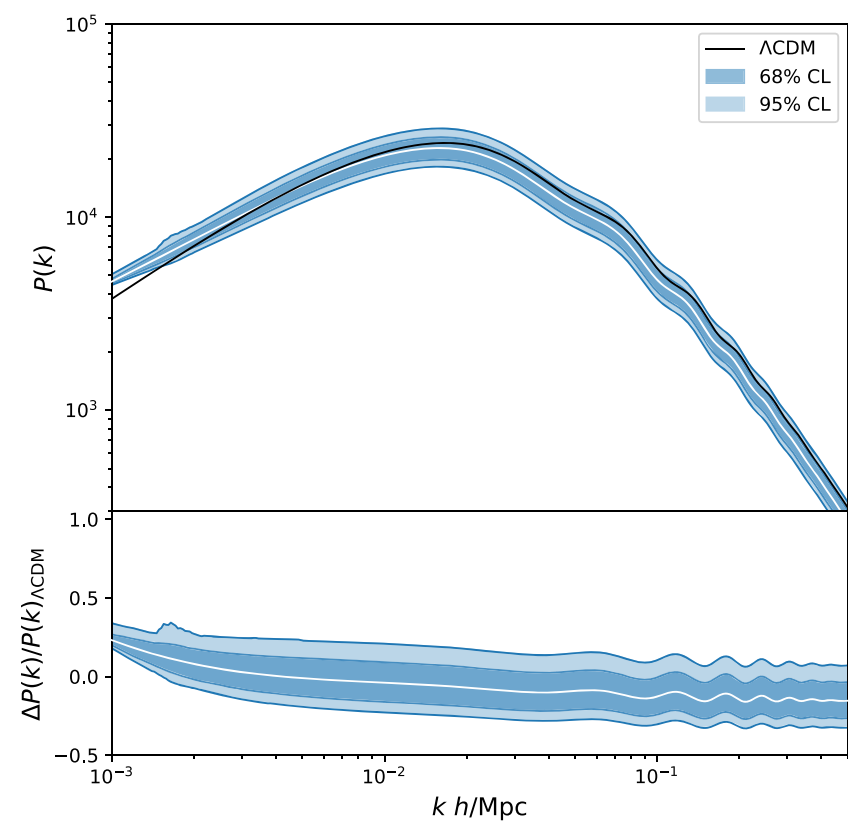

FIG. 10. The matter power spectrum distribution for model 2 theories reconstructed using the H2F expansion history. The bestfit $\Lambda \mathrm{CDM}$ spectrum is shown for reference. Distribution of spectra for $\mathrm{H} 1, \mathrm{H} 1 \mathrm{~F}$ and $\mathrm{H} 2$ are very similar.

spectrum. As in the case of the CMB spectrum, we expect that this difference can accommodated by adjusting other parameters in a comprehensive fit which, however, is beyond the scope of this work. In addition to the change in the matter-DE equality, $P(k)$ is also effected by the larger $G_{\text {eff }}$ and the fifth force mediated by the scalar field. This is encoded in the way the deviations from $\Lambda \mathrm{CDM}$ increase on smaller scales. Finally the oscillations that we note at $k \approx$ $0.1 \mathrm{~h} / \mathrm{Mpc}$ are due to the different position of the BAO scale due to a slightly different expansion history of the GBD models.

In Fig. 7 we show the theoretical prediction of the crosscorrelations for the two classes of reconstructed GBD theories from $\mathrm{H} 2 \mathrm{~F}$. Also shown is the cosmic variance statistical uncertainty in the cross-correlation predicted by the $\Lambda \mathrm{CDM}$ model. As one can see, in some model 1 theories, the ISW effect can become negative signaling a growing gravitational potential due to the fifth force mediated by the extra scalar field. At lower redshifts, when the effective DE becomes larger and the growth of the gravitational potential is overcome by the decay induced by the accelerated expansion, the ISW term is mainly positive.

Some of the model 1 theories reconstructed from the $\mathrm{H} 2 \mathrm{~F}$ expansion history are cosmologically viable, at least from the perspective of fitting the CMB spectra. Figure 8 shows the cosmological observables for the four representative models whose reconstructed potentials $U(\phi)$ were shown in Fig. 3. While the CMB anisotropies are almost the same for each model, they differ considerably in the clustering of matter and this is also noticeable in the crosscorrelations $C_{\ell}^{T g}$ at the bottom panels. In the higher redshift windows, the larger values of the couplings constant $\xi$ drive a growth of the gravitational potentials $\Psi$ and $\Phi$ due to the fifth force mediated by the scalar field, causing a negative ISW effect. When DE eventually starts dominating the potentials stop growing and instead decay, turning the sign of the ISW effect.

\section{B. Observables for model 2}

Figure 9 shows the distribution of the CMB anisotropy spectra corresponding to the sampled model 2 theories reconstructed from the $\mathrm{H} 1, \mathrm{H} 1 \mathrm{~F}, \mathrm{H} 2$ and $\mathrm{H} 2 \mathrm{~F}$ expansion histories. We see in all cases there is a preference for a large ISW contribution to $\mathcal{D}_{\ell}$. This is especially the case for $\mathrm{H} 1$, in which $X(a)$ is nonmonotonic and has a large increase. However, since cosmic variance results in large statistical error bars at small $\ell$, there are models on the fringe of the allowed range for $\mathrm{H} 1 \mathrm{~F}, \mathrm{H} 2$ and $\mathrm{H} 2 \mathrm{~F}$ that can be compatible with the current data.

The model 2 distribution of $P(k)$ is in good agreement with the data, with the best-fit $\Lambda \mathrm{CDM}$ prediction being well inside the 68\% CL, as shown in Fig. 10 in the case of H2F. The matter power spectra in the cases of $\mathrm{H} 1, \mathrm{H} 1 \mathrm{~F}$ and $\mathrm{H} 2 \mathrm{~F}$ are very similar.

A very distinctive observational feature of model 2 models is a large positive ISW signal at high redshifts, as seen in Fig. 11. This is caused by $F>1$ at $z \gtrsim 1$, which decreases the effective Newton's constant appearing in the Poisson equation $\left(G_{\text {eff }} \propto G / F\right)$ resulting in a suppression
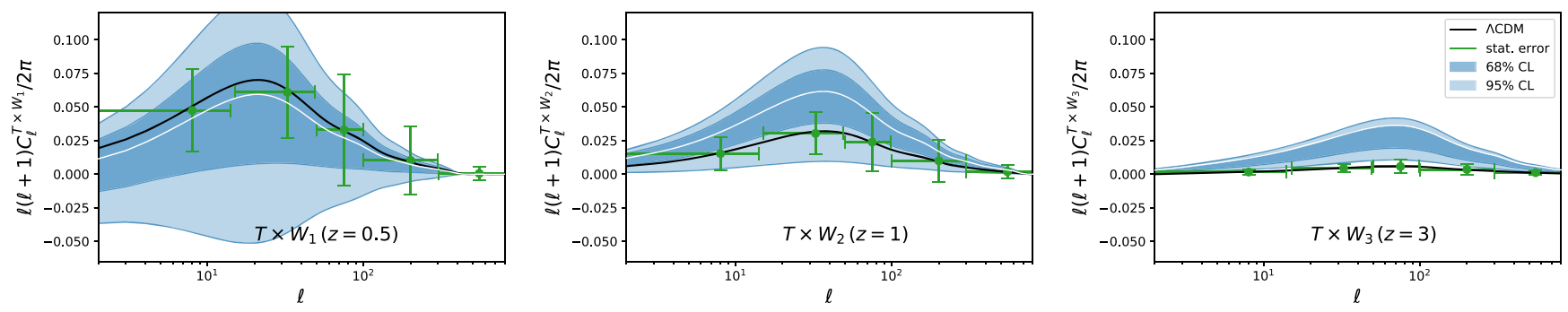

FIG. 11. The distribution of CMB temperature and GNC cross-correlation spectra for model 2 theories reconstructed using the H2F expansion history. The best-fit $\Lambda \mathrm{CDM}$ spectra, along with the statistical error bars, are shown for reference. The distribution of spectra for $\mathrm{H} 1, \mathrm{H} 1 \mathrm{~F}$ and $\mathrm{H} 2$ are qualitatively similar. 

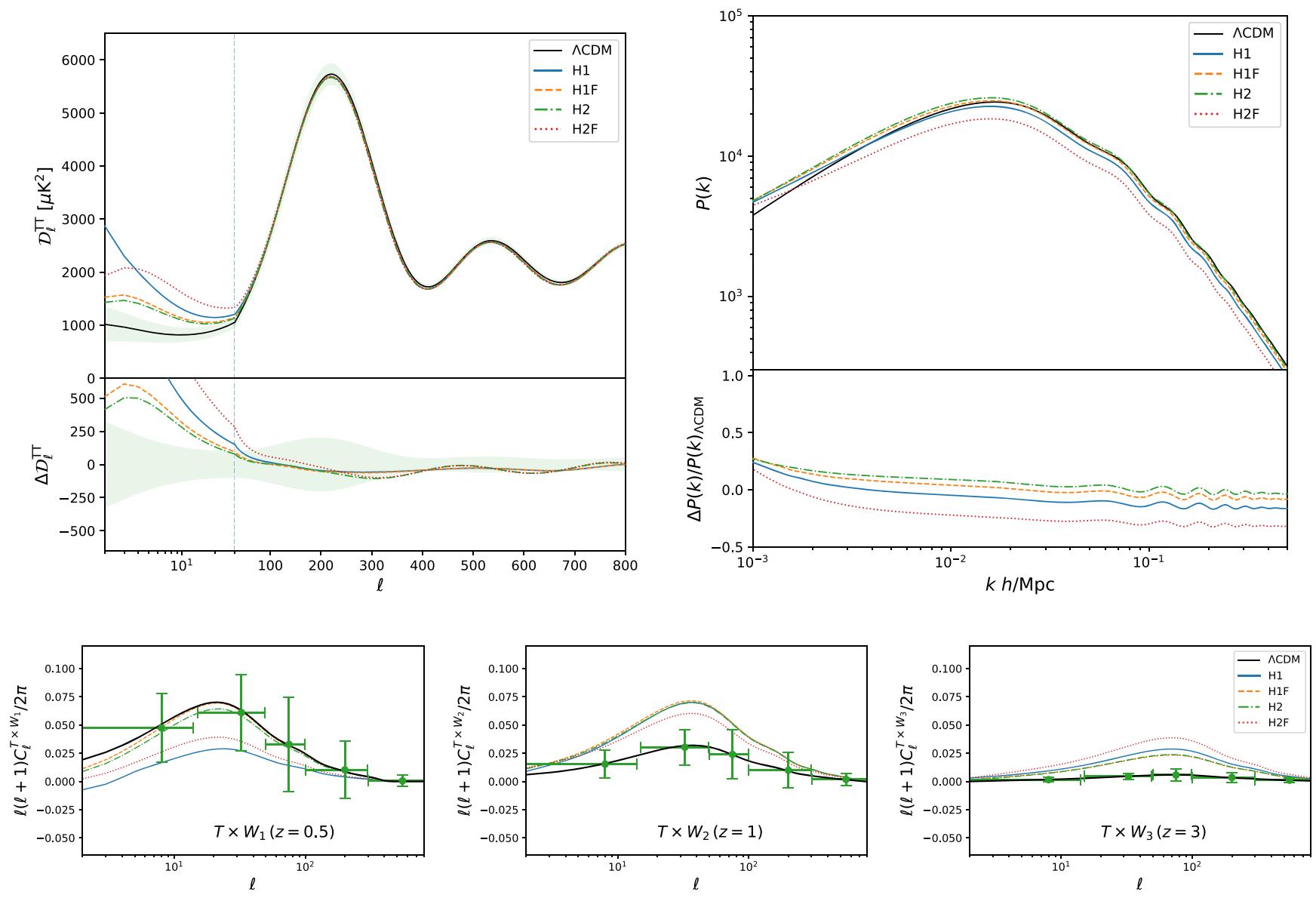

FIG. 12. Cosmological observables in four representative model 2 theories reconstructed from $\mathrm{H} 1, \mathrm{H} 1 \mathrm{~F}, \mathrm{H} 2$ and $\mathrm{H} 2 \mathrm{~F}$ expansion histories. The best-fit $\Lambda \mathrm{CDM} C \mathrm{CMB}$ spectrum, along with the statistical error bars, is shown for reference.

of gravitational potentials during the matter-dominated epoch. The enhancement in the high redshift crosscorrelation is well in excess of the cosmic variance uncertainty around the $\Lambda \mathrm{CDM}$ model and would be detectable with the next-generation large-scale structure surveys such as DESI, LSST and Euclid. We note that recent redshift space distortion measurements slightly favor a lower value of $G_{\text {eff }}$ [99].

In Fig. 12 we show observables corresponding to the four models in Fig. 5 representing reconstructions using $\mathrm{H} 1, \mathrm{H} 1 \mathrm{~F}, \mathrm{H} 2$ and H2F. We can see the similarity in general trends, with features being the most pronounces in the case of $\mathrm{H} 1$ and less so for H2F. However, in all cases, there is a large positive ISW signal at high redshifts which would be a smoking gun of GBD models with a nonmonotonic $F(\phi)$.

\section{CONCLUSIONS}

Current observations favor an increasing effective DE density, corresponding to an effective DE EOS that is less than -1 [27,28]. Such apparently phantom behavior of DE can also occur in GBD theories, as a manifestation of the additional interaction mediated by the scalar field.
We have set up a reconstruction method to design the Lagrangians of GBD-type scalar-tensor theories corresponding to expansion histories extracted using the latest data probing the background [28]. We then examined the viability of such designer GBD theories, both in terms of their stability and their ability to predict acceptable cosmological observables.

We found that a large increase in the effective DE density, or the apparent oscillatory dynamics also favored by the data, are difficult to accommodate within a GBD theory with a monotonically evolving coupling function, such as $F(\phi) \propto \exp (\xi \phi)$. However, allowing for an arbitrary $F(a)$, parametrized in terms of a polynomial expansion, results in GBD theories capable of fitting current $\mathrm{CMB}$ and matter power spectra.

We find that, in viable models, $F(a)$ increases at high redshifts before decreasing at more recent epochs, leading to a smaller effective gravitational coupling $G_{\text {eff }}$ at redshifts $z \gtrsim 1$ and a larger $G_{\text {eff }}$ at $z<1$. This leads to a robust prediction of a large positive ISW signal at $z>1$, which would be readily detectable through CMB-galaxy crosscorrelation using high redshift sources from DESI, LSST and Euclid. 
In our analysis, we opted to provide functions $F(\phi)$ or $F(a)$ and reconstruct the potential $U(\phi)$. One could, alternatively, opt to find $F(\phi)$ for a given $U(\phi)$. We expect that, regardless of the choice, the main conclusion about the key role of the ISW effect in falsifying GBD theories will remain the same.

The method developed here is complementary to the reconstruction of the EFT functions (including the GBD subset of the EFTofDE) from cosmological observations performed in [100]. In that work, the expansion history was reconstructed in conjunction with the scalar-tensor Lagrangian, thus only producing expansion histories that are consistent with the GBD. Our approach is different-we start with an expansion history obtained from the data in a largely model-independent way and checked if there can be GBD theories producing it. The difference is that a joint reconstruction within the GBD framework could miss expansion histories that are difficult to accommodate with smooth EFT functions, potentially missing a hint for dynamics that would correspond to a rare realization of GBD.

Our results show that one could rule out scalar-tensor theories as the explanation of departures from the $\Lambda \mathrm{CDM}$ background expansion history using purely cosmological datasets. This is particularly important for testing theories in which the scalar field couples only to dark matter, to which the tight laboratory and Solar System tests of gravity do not apply.

\section{ACKNOWLEDGMENTS}

We acknowledge G. Papadomanolakis and S. Peirone for useful discussions and feedback on our findings. A. Z. and L. P. are supported by the Natural Sciences and Engineering Research Council (NSERC) of Canada. A. S. acknowledges support from the NWO and the Dutch Ministry of Education, Culture and Science (OCW), and from the D-ITP consortium, a program of the NWO that is funded by the OCW. G. B.Z. is supported by the National Key Basic Research and Development Program of China (No. 2018YFA0404503) and by NSFC Grants No. 11720101004 and No. 11673025. Y. W. is supported by the Nebula Talents Program of National Astronomy Observatories of China. This research was enabled in part by support provided by WestGrid and Compute Canada.
[1] S. Perlmutter et al. (Supernova Cosmology Project), Astrophys. J. 517, 565 (1999).

[2] A. G. Riess et al. (Supernova Search Team), Astron. J. 116, 1009 (1998).

[3] C. L. Bennett et al., Astron. Astrophys. Suppl. Ser. 208, 20 (2013).

[4] P. A. R. Ade et al. (Planck Collaboration), Astron. Astrophys. 594, A13 (2016).

[5] W. J. Percival et al. (2dFGRS Team), Mon. Not. R. Astron. Soc. 337, 1068 (2002).

[6] F. Beutler, C. Blake, M. Colless, D. H. Jones, L. StaveleySmith, L. Campbell, Q. Parker, W. Saunders, and F. Watson, Mon. Not. R. Astron. Soc. 416, 3017 (2011).

[7] S. Alam et al. (BOSS Collaboration), Mon. Not. R. Astron. Soc. 470, 2617 (2017).

[8] A. Conley et al. (SNLS Collaboration), Astron. Astrophys. Suppl. Ser. 192, 1 (2011).

[9] N. Suzuki et al., Astrophys. J. 746, 85 (2012).

[10] I. Zehavi, Z. Zheng, D. H. Weinberg, M. R. Blanton, N. A. Bahcall, A. A. Berlind, J. Brinkmann, J. A. Frieman, J. E. Gunn, R. H. Lupton, R. C. Nichol, W. J. Percival, D. P. Schneider, R. A. Skibba, M. A. Strauss, M. Tegmark, and D. G. York, Astrophys. J. 736, 59 (2011).

[11] C. Heymans et al., Mon. Not. R. Astron. Soc. 427, 146 (2012).

[12] H. Hildebrandt et al., Mon. Not. R. Astron. Soc. 465, 1454 (2017).

[13] C. P. Burgess, arXiv:1711.10592.

[14] A. G. Riess et al., Astrophys. J. 826, 56 (2016).
[15] A. G. Riess, S. Casertano, W. Yuan, L. M. Macri, and D. Scolnic, Astrophys. J. 876, 85 (2019).

[16] T. M. C. Abbott et al. (DES Collaboration), Phys. Rev. D 98, 043526 (2018).

[17] K. C. Wong et al., arXiv:1907.04869.

[18] D. Scott, arXiv:1804.01318.

[19] C. Dvorkin, M. Wyman, D. H. Rudd, and W. Hu, Phys. Rev. D 90, 083503 (2014).

[20] E. Di Valentino, A. Melchiorri, and J. Silk, Phys. Lett. B 761, 242 (2016).

[21] E. Di Valentino, C. Boehm, E. Hivon, and F. R. Bouchet, Phys. Rev. D 97, 043513 (2018).

[22] E. Di Valentino, E. V. Linder, and A. Melchiorri, Phys. Rev. D 97, 043528 (2018).

[23] S. Adhikari and D. Huterer, arXiv:1905.02278.

[24] S. Alexander and E. McDonough, Phys. Lett. B 797, 134830 (2019).

[25] M.-X. Lin, G. Benevento, W. Hu, and M. Raveri, Phys. Rev. D 100, 063542 (2019).

[26] S. Peirone, G. Benevento, N. Frusciante, and S. Tsujikawa, Phys. Rev. D 100, 063540 (2019).

[27] G.-B. Zhao et al., Nat. Astron. 1, 627 (2017).

[28] Y. Wang, L. Pogosian, G.-B. Zhao, and A. Zucca, Astrophys. J. 869, L8 (2018).

[29] W. Yang, A. Mukherjee, E. Di Valentino, and S. Pan, Phys. Rev. D 98, 123527 (2018).

[30] E. Di Valentino, R. Z. Ferreira, L. Visinelli, and U. Danielsson, Phys. Dark Universe 26, 100385 (2019).

[31] R. R. Caldwell, Phys. Lett. B 545, 23 (2002). 
[32] S. M. Carroll, M. Hoffman, and M. Trodden, Phys. Rev. D 68, 023509 (2003).

[33] A. Vikman, Phys. Rev. D 71, 023515 (2005).

[34] U. Alam, V. Sahni, T. D. Saini, and A. A. Starobinsky, Mon. Not. R. Astron. Soc. 354, 275 (2004).

[35] T. Holsclaw, U. Alam, B. Sanso, H. Lee, K. Heitmann, S. Habib, and D. Higdon, Phys. Rev. Lett. 105, 241302 (2010).

[36] S. Das, P. S. Corasaniti, and J. Khoury, Phys. Rev. D 73, 083509 (2006).

[37] R. Gannouji, D. Polarski, A. Ranquet, and A. A. Starobinsky, J. Cosmol. Astropart. Phys. 09 (2006) 016.

[38] L. Amendola and S. Tsujikawa, Phys. Lett. B 660, 125 (2008).

[39] R. G. Crittenden and N. Turok, Phys. Rev. Lett. 76, 575 (1996).

[40] N. Afshordi, Phys. Rev. D 70, 083536 (2004).

[41] T. Giannantonio, R. Scranton, R. G. Crittenden, R. C. Nichol, S. P. Boughn, A. D. Myers, and G. T. Richards, Phys. Rev. D 77, 123520 (2008).

[42] A. J. Shajib and E. L. Wright, Astrophys. J. 827, 116 (2016).

[43] L. Pogosian, J. Cosmol. Astropart. Phys. 04 (2005) 015.

[44] L. Pogosian, P. S. Corasaniti, C. Stephan-Otto, R. Crittenden, and R. Nichol, Phys. Rev. D 72, 103519 (2005).

[45] Y.-S. Song, W. Hu, and I. Sawicki, Phys. Rev. D 75, 044004 (2007).

[46] J. Renk, M. Zumalacarregui, and F. Montanari, J. Cosmol. Astropart. Phys. 07 (2016) 040.

[47] A. Aghamousa, J. Aguilar, S. Ahlen, S. Alam, L. E. Allen, C. Allende Prieto, J. Annis, S. Bailey, C. Balland et al. (DESI Collaboration), arXiv:1611.00036.

[48] v. Ivezić et al. (LSST Collaboration), Astrophys. J. 873, 111 (2019).

[49] P. A. Abell et al. (LSST Collaboration), arXiv:0912.0201.

[50] A. Refregier (EUCLID Collaboration), Exp. Astron. 23, 17 (2009).

[51] A. Cimatti et al. (SPACE Team), Exp. Astron. 23, 39 (2009).

[52] R. Laureijs, arXiv:0912.0914.

[53] R. G. Crittenden, G.-B. Zhao, L. Pogosian, L. Samushia, and X. Zhang, J. Cosmol. Astropart. Phys. 02 (2012) 048.

[54] R. G. Crittenden, L. Pogosian, and G.-B. Zhao, J. Cosmol. Astropart. Phys. 12 (2009) 025.

[55] A. Silvestri and M. Trodden, Rep. Prog. Phys. 72, 096901 (2009).

[56] T. Clifton, P. G. Ferreira, A. Padilla, and C. Skordis, Phys. Rep. 513, 1 (2012).

[57] M. Betoule et al. (SDSS Collaboration), Astron. Astrophys. 568, A22 (2014).

[58] M. Moresco, L. Pozzetti, A. Cimatti, R. Jimenez, C. Maraston, L. Verde, D. Thomas, A. Citro, R. Tojeiro, and D. Wilkinson, J. Cosmol. Astropart. Phys. 05 (2016) 014.

[59] A. J. Ross, L. Samushia, C. Howlett, W. J. Percival, A. Burden, and M. Manera, Mon. Not. R. Astron. Soc. 449, 835 (2015).

[60] G.-B. Zhao et al. (BOSS Collaboration), Mon. Not. R. Astron. Soc. 466, 762 (2017).
[61] Y. Wang et al. (BOSS Collaboration), Mon. Not. R. Astron. Soc. 469, 3762 (2017).

[62] M. Ata et al., Mon. Not. R. Astron. Soc. 473, 4773 (2018).

[63] A. Font-Ribera et al. (BOSS Collaboration), J. Cosmol. Astropart. Phys. 05 (2014) 027.

[64] T. Delubac et al. (BOSS Collaboration), Astron. Astrophys. 574, A59 (2015).

[65] V. de Sainte Agathe et al., Astron. Astrophys. 629, A85 (2019).

[66] M. Blomqvist et al., Astron. Astrophys. 629, A86 (2019).

[67] G.-B. Zhao, R. G. Crittenden, L. Pogosian, and X. Zhang, Phys. Rev. Lett. 109, 171301 (2012).

[68] P. G. Bergmann, Int. J. Theor. Phys. 1, 25 (1968).

[69] K. Nordtvedt, Jr, Astrophys. J. 161, 1059 (1970).

[70] R. V. Wagoner, Phys. Rev. D 1, 3209 (1970).

[71] A. Lewis, A. Challinor, and A. Lasenby, Astrophys. J. 538, 473 (2000).

[72] S. M. Carroll, A. De Felice, and M. Trodden, Phys. Rev. D 71, 023525 (2005).

[73] V. Sahni and Y. Shtanov, J. Cosmol. Astropart. Phys. 11 (2003) 014.

[74] U. Alam, S. Bag, and V. Sahni, Phys. Rev. D 95, 023524 (2017).

[75] B. Boisseau, G. Esposito-Farese, D. Polarski, and A. A. Starobinsky, Phys. Rev. Lett. 85, 2236 (2000).

[76] G. Esposito-Farese and D. Polarski, Phys. Rev. D 63, 063504 (2001).

[77] L. Perivolaropoulos, J. Cosmol. Astropart. Phys. 10 (2005) 001.

[78] L. Pogosian and A. Silvestri, Phys. Rev. D 77, 023503 (2008); 81, 049901(E) (2010).

[79] J.-P. Uzan, Living Rev. Relativity 14, 2 (2011).

[80] J. Khoury and A. Weltman, Phys. Rev. Lett. 93, 171104 (2004).

[81] K. Hinterbichler and J. Khoury, Phys. Rev. Lett. 104, 231301 (2010).

[82] B. Hu, M. Raveri, N. Frusciante, and A. Silvestri, Phys. Rev. D 89, 103530 (2014).

[83] M. Raveri, B. Hu, N. Frusciante, and A. Silvestri, Phys. Rev. D 90, 043513 (2014).

[84] N. Frusciante, G. Papadomanolakis, S. Peirone, and A. Silvestri, J. Cosmol. Astropart. Phys. 02 (2019) 029.

[85] A. De Felice, N. Frusciante, and G. Papadomanolakis, J. Cosmol. Astropart. Phys. 03 (2017) 027.

[86] A. E. Gumrukcuoglu, S. Mukohyama, and T. P. Sotiriou, Phys. Rev. D 94, 064001 (2016).

[87] M. Zumalacrregui, E. Bellini, I. Sawicki, and J. Lesgourgues, J. Cosmol. Astropart. Phys. 08 (2017) 019.

[88] J. Khoury and A. Weltman, Phys. Rev. D 69, 044026 (2004).

[89] P. Brax, C. van de Bruck, A.-C. Davis, J. Khoury, and A. Weltman, Phys. Rev. D 70, 123518 (2004).

[90] B. Hu, M. Raveri, N. Frusciante, and A. Silvestri, arXiv: 1405.3590.

[91] https://github.com/EFTCAMB/EFTCAMB.

[92] G. Gubitosi, F. Piazza, and F. Vernizzi, J. Cosmol. Astropart. Phys. 02 (2013) 032. 
[93] J. K. Bloomfield, É. É. Flanagan, M. Park, and S. Watson, J. Cosmol. Astropart. Phys. 08 (2013) 010.

[94] A. Challinor and A. Lewis, Phys. Rev. D 84, 043516 (2011).

[95] https://github.com/cmbant/CAMB/tree/CAMB_sources.

[96] A. Lewis and A. Challinor, Phys. Rev. D 76, 083005 (2007).
[97] P. A. R. Ade et al. (Planck Collaboration), Astron. Astrophys. 594, A21 (2016).

[98] Y. Akrami et al. (Planck Collaboration), arXiv:1807. 06205.

[99] G.-B. Zhao et al., Mon. Not. R. Astron. Soc. 482, 3497 (2019).

[100] M. Raveri, arXiv:1902.01366. 